\title{
A model for the Guaraní expansion in the La Plata Basin and littoral zone of southern Brazil
}

\author{
Mariano Bonomo a, *, Rodrigo Costa Angrizani a, Eduardo Apolinaire a , \\ Francisco Silva Noelli ${ }^{\mathrm{b}}$ \\ ${ }^{a}$ CONICET-Universidad Nacional de La Plata, Facultad de Ciencias Naturales y Museo, Museo de La Plata, División Arqueología, Paseo del Bosque, s/n ${ }^{\circ}$ \\ 1900 La Plata, Buenos Aires Province, Argentina \\ ${ }^{\mathrm{b}}$ Universidade Estadual de Maringá, Brazil
}

\section{A R T I C L E I N F O}

\section{Article history:}

Available online 8 December 2014

\section{Keywords}

Guaraní archaeology

South American Lowlands

Late Holocene

GIS

Radiocarbon and thermoluminescence dates

\begin{abstract}
A B S T R A C T
Since the 19th century, it has been proposed that the wide geographic distribution of the Guaraní indigenous populations in large areas of South America was the result of prehistoric movements of large numbers of people through the main rivers of the La Plata Basin and the Brazilian coastline. However, no detailed spatial and temporal analysis has been carried out with the dated Guarani archaeological sites of the different zones of Brazil, Argentina, and Uruguay. This is one of the reasons why some of the main ideas about this past population expansion still lack empirical validation. This paper presents a study on the geographic and temporal distribution of the Guaraní archaeological sites in the La Plata Basin and the Atlantic coast of southern Brazil. The overall purpose is to contribute to understanding the Guarani expansion using the analysis of two basic archaeological variables: space and time. Based on available numerical dates $\left({ }^{14} \mathrm{C}\right.$ and thermoluminescence) and the application of GIS software, potential dispersion routes are discussed. To date, more than 1100 archaeological sites assigned to the Guaraní, along with almost 250 numerical dates were analyzed. With this data, it was possible to elaborate a Dispersion Routes Model and a Temporal Visualization Model, which enabled identification of the sequence of population dispersal, with two main expansion pulses, throughout nearly two millennia of Guaraní movements across the South American Lowlands.
\end{abstract}

(C) 2014 Elsevier Ltd and INQUA. All rights reserved.

\section{Introduction}

The indigenous populations of the Tupí linguistic stock are widespread throughout the South American Lowlands. In spite of the thousands of kilometers separating them, they share numerous cultural and linguistic features due to their common ancestral origin. Together, there are around sixty languages grouped in ten linguistic families: Tupí-Guaraní, Awetí, Mawé, Mundurukú, Jurúna, Arikém, Tuparí, Ramaráma, Mondé and Puruborá (Rodrigues and Cabral, 2012). The Tupí-Guaraní family, which includes the Ancient Guaraní and the Tupinambá, among its forty languages, is the largest and the most broadly spatially dispersed. At the moment of European conquest in the 16th century, the populations speaking the languages of this family extended almost without interruption along the Brazilian littoral zone and in many

\footnotetext{
* Corresponding author.

E-mail addresses: mbonomo@fcnym.unlp.edu.ar (M. Bonomo), rcangrizani@ gmail.com (R. Costa Angrizani), eapolinaire@gmail.com (E. Apolinaire), ffnoelli@ wnet.com.br (F.S. Noelli).
}

inland regions. They occupied, as in the present, different areas, from the Amazon basin to the eastern Chaco and the Andean foothills. Within this linguistic family, the speakers of the Ancient Guarani language were found in a portion of the Atlantic coast, south of Paranaguá in southern Brazil, in parts of Uruguay, and in the surroundings of the La Plata River. Inland, they inhabited the margins of some watercourses draining directly to the ocean and large areas of the Paraguay, Paraná and Uruguay Basins, including the ancient Guairá (or Guayrá) and the Misiones historic regions. Accordingly, this vast geographic distribution has been seen as an indicator of a long history of repetitive movement of people over long distances, which persisted in the form of the recent migrations recorded after the European arrival (Métraux, 1927; Brochado, 1984).

The interest in providing a scientific explanation for the wide dispersion of populations belonging to the Tupí stock is not new. Since the 19th century, many researchers had begun to search their Amazonian centre of origin and the prehistoric routes followed during their dispersal (Rodrigues, 1964, 1986; Brochado, 1984; Meggers et al., 1988; Urban, 1992; Heckenberger et al., 1998; 
Noelli, 1998); issues which are still subject to debate. Given the complexity of the Tupi phenomenon, we limited both thematically and spatially to the Guaraní archaeological record. According to the abundant historical accounts, Guaraní people lived in riverside palisade villages. They based their economy on horticulture (principally corn and manioc), as well as hunting, gathering, and fishing. They were excellent navigators that traveled by canoe along the long river routes of La Plata Basin and adjacent regions. Traditionally, historic Guaraní people and their immediate prehistoric ancestors have been identified in the archaeological record based on the occurrence of the following features: 1) ceramic dishes, shallow bowls and large jars (mainly restricted orifice, conical base and complex profiles with angle and inflection points), 2) corrugated surface treatments of the vessels, in addition to nail-incised, brushed or painted (red and/or black lines over white slip), 3) lip plugs named tembetás, 4) polished-stone axes, 5) secondary burials in urns and/or 6) bounded dark sediments named patches of terra preta sediment, associated with households and other architectural structures.

This article is focused on the study of the spatial and temporal distribution of the archaeological sites attributed to the Guaraní in the La Plata Basin and littoral zone of southern Brazil. The overall objective of this paper is to analyze continuous Guaraní expansion throughout the main waterways and the Atlantic coastline. In order to attain this objective, an intense review of the ${ }^{14} \mathrm{C}$ and thermoluminescence available dates of Guaraní sites was carried out. Then, a Geographic Information System (GIS) database was created to correlate the location and chronology of the recorded sites; resulting in temporal maps, modeling of the Guaraní dispersion routes, and the identification of major expansion pulses. Finally, the expansion model was compared with the previous expansion model proposed by Brochado's (1984).

\section{The scientific study of Guaraní archaeology}

In order to address the Guaraní archaeological record, it is necessary to introduce some comments to contextualize our study. First, although it is obviously incorrect to claim that there is always a direct relationship between particular archaeological materials and specific ethnic group (or language) (see Soares, 2001-2002, Farías Gluchy, 2005; Lima, 2011 for the Guaraní), it is also erroneous to believe the opposite and dismiss the links between the material culture and the people who produced it (Brochado, 1984; Anthony, 2010). The similar village-settlement and economic patterns, the technological, functional and stylistic recurrences in the vessels, and the uniformity in the treatment of the dead have all enabled the conformation of a distinctive archaeological entity which generally coincides with the distribution that the Guarani speakers had five centuries ago (Nimuendajú, 1982; Urban, 1992). The Guaraní archaeological entity is used here, but in doing so, there is no intention to defend the mechanical relation between isolated traits and static ethnic groups. Ethnicity is considered as a process recognizable on the observable patterns of the material culture (Hodder, 1979), especially in cases like the Guaraní, which comprises of large samples of historical, ethnographic, linguistic, physical anthropology, and archaeological data. This strongly validates these associations among common and recurring elements throughout a considerable period of time, and in areas where were not previously found.

Secondly, the most appropriate approaches to the Guaraní archaeology must include the interaction among the different information generated by different disciplines studying the same people. Linguistics, physical anthropology, and genetics can offer a compelling view on the origin of populations of different linguistic families and their pattern of dispersion, and it can help archaeology to understand the complex relation between people, language, and material culture (eg. Neves et al., 2011; Ramallo et al., 2013; see discussion in Anthony, 2010). Ethnology and ethnohistory, along with archaeology, can be useful to define the material culture, its lexicon, and technological styles, along with the indigenous economies and strategies used for territorial dominance. Finally, ethnoarchaeology and historical ecology offer insights into the traditional knowledge, in order to research how indigenous people reproduce their culture and adapt to their environment by transforming it into humanized landscapes (Silva, 2008; Balée, 2013). Therefore, this interdisciplinary and comprehensive perspective of archaeology, as opposed to a unilateral one, not only provides more empirical data, but also offers new viewpoints for the construction of the Guaraní knowledge. This allows a more concrete perception of the different regional processes, along with a more precise definition of the material culture and the interaction with other people. With time, this will enable the development of better conditions to understand and compare the Guaraní archaeological record and systemic contexts.

The presence of archaeological contexts assigned to the Guaraní in the La Plata Basin and in southern Brazil has been investigated by numerous researchers since the 19th century (Ambrosetti, 1895; Lothrop, 1932; Menghín, 1956; Chmyz, 1968; Pronapa, 1970; Cigliano et al., 1971; Meggers and Evans, 1983; Brochado, 1984; Caggiano, 1984; Scatamacchia, 1990; Schmitz, 1991; Cabrera Pérez, 1994; Sempé and Caggiano 1995; Noelli, 1998; Rogge, 2005; Kashimoto and Martins, 2008; Milheira, 2008; Prous and Lima, 2008; Rodríguez, 2009; Loponte et al., 2011; Costa Angrizani, 2012; Bonomo, 2013; Noelli et al., 2014; among many others). Many researchers included these archeological contexts in the Tupiguaraní Tradition defined for Brazil (Chmyz, 1966; Pronapa, 1970). This tradition comprises the archaeological manifestations of polychrome, corrugated, or brushed pottery, tembetás, stone axes, and secondary urn burials. Inspired by Willey and Phillips (1958), the main objectives of this classification was to arrange in both time and space different sub-traditions and phases which had been defined through the variations in the temper material and surface treatment of pottery fragment assemblages (Evans and Meggers, 1965; Meggers and Evans, 1970), disregarding aspects such as vessel shapes or functions. More than seventy phases were proposed for the Guaraní area. These phases were defined by variable combinations of sherd surface treatments (Evans and Meggers, 1965), collected under questionable statistical criteria, lack of stratigraphic control in several excavations, and insufficient numerical dates (Dias, 1995; Noelli, 1998, 2008). In spite of being adopted, even today, by archaeologists from Brazil, Uruguay, and Argentina, the Tupiguaraní Tradition has received several criticism and revisions since the 1980s (Brochado, 1984; Dias, 1995; Noelli, 2008). Basically, this scheme was questioned for being too broad and arbitrarily compressing under the same archaeological category, the material culture (especially the pottery) of different Tupí speaking people or other cultural traditions. Moreover this line of thought was indifferent to the incorporation of ethnohistoric or ethnographic data, and thus failed to generate conceptual tools or methodologies to deeply investigate the historical processes throughout their complete duration.

The shift of perspectives started in the 1970s, when Donald Lathrap inaugurated a new interdisciplinary line of research for Amazonian archaeology (Lathrap, 1970; Oliver, 1991, 2008). He adopted the linguistic hypothesis of Aryon Rodrigues -who had begun to demonstrate the genetic relations among languages, define families, and order the Tupí stock-, thus enabling a course to study past geographic dispersion processes. According to linguistic research, this stock had its origin in the Proto-Tupí ancestral tongue, dating back to around four or five thousand years ago 
(Urban, 1992; Storto and Moore, 2001; Rodrigues and Cabral, 2012). The study of languages through the historic-comparative method paved the way for the reconstruction of the original Proto-Tupí sounds, lexicon and part of the grammar of this mother tongue (Correa da Silva, 2010). Two significant aspects were proposed through this method: the existence of pottery and agriculture at the time when the Proto-Tupí language emerged (Rodrigues, 2007a,b, 2010). The acknowledgement of the Proto-Tupí and the genetic criteria shared by the languages comprising the stock families provided a scientific model through which it was possible to start explaining the patterns of material culture shared by them. Based on the common origin hypothesis, meaning that the different languages within a family are manifestations of the same original language altered by time (Rodrigues, 1986:29), it was possible to explain the similarities found among surface treatments and vessel profiles in the Tupí pottery. This had been already indicated by Alfred Métraux (1928, for a statistical analysis of Métraux's data cf. Klimek and Mielke, 1935), who exposed similarities among other materials, in addition to their labels and functions.

The theoretical development and the empirical support of many of Lathrap's ideas were prepared by José Brochado (Noelli, 2008). His PhD thesis is one of the most comprehensive and inspiring text written on the Guaraní archaeology record. Brochado (1984:1) begins his thesis by defending the attempts to determine the continuity processes between the systemic and archaeological contexts. He utilized diverse methodologies in order to integrate historical, ethnographic, linguistic, and archaeological data (Brochado, 1977, 1984, 1991; La Salvia and Brochado, 1989). Brochado's hypothesis was that the Proto-Tupí speakers created a pottery style which would be reproduced throughout time, but which would also transform following the changes occurring in the genetic derivation of the languages. In that way, it was possible to conceive that each group speaking one of the Tupí variants would have its own artefactual assemblage, distinguished from the others' by some subtle uniqueness. With the aim of showing the similarities and variations in the Tupí pottery, Brochado studied in great detail a large quantity of archaeological artifacts from the Guaraní and the Tupinambá (distributed manly along the Atlantic coast between the Brazilian state of Paraná and the mouth of the Amazon River); Tupinambá and Guaraní cranial morphology shows strong association (Neves et al., 2011), and their languages belonging to the Tupí-Guaraní linguistic family, which were separated 2500 years ago (Rodrigues, 1964). Among his remarkable contributions, the most outstanding one was that he provided archaeologists with historical data about an emic lexicon for the Guaraní pottery, based in its shapes and functions. Brochado proposed the existence of different vessel types which could be defined according to the combinations of their temper, surface treatment and pot zones or segments (La Salvia and Brochado, 1989:116).

Throughout its whole area of occurrence, Guaraní pottery (Fig. 1) shows a high level of formal standardization, following strict rules of proportion, surface treatment, and decoration; thus attesting the conservation of the traditional knowledge throughout long time periods and across wide distances (La Salvia and Brochado, 1989; Brochado et al., 1990; Brochado and Monticelli, 1994; Noelli and Brochado, 1998). The pottery presents distinct characteristics compared to pottery made by other non-Tupí groups in the La Plata Basin. When considered as a whole, these pottery assemblages serve as a good indicator for the past Guaraní settlements. Notwithstanding this, on the basin of the Paranapanema

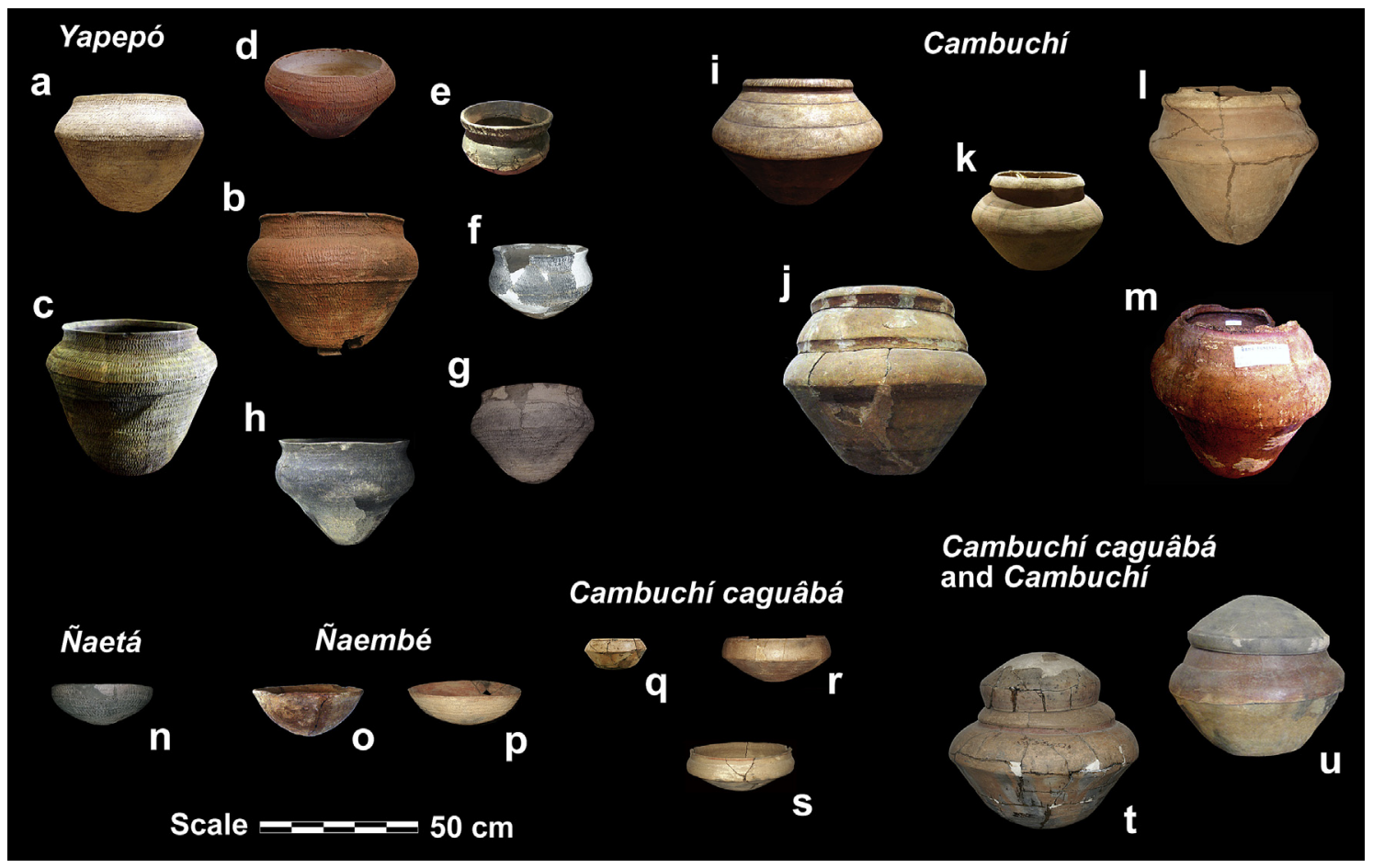

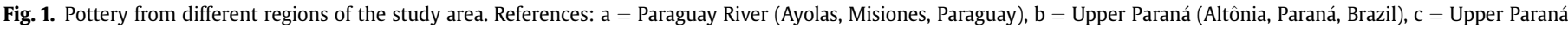

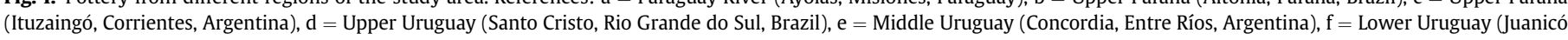

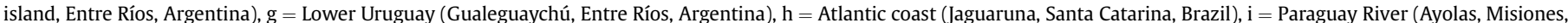

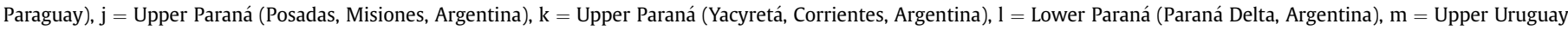

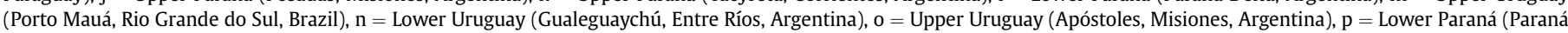

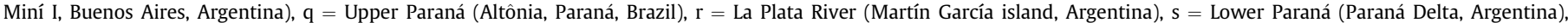

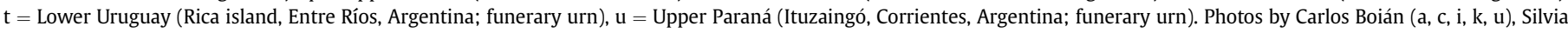
Jordán (e, j) and Ulises Nahirñak (o). 
River, in the Brazilian state of São Paulo, and the northeast of the state of Mato Grosso do Sul, the only way to differentiate Guarani pottery from Tupinambá pottery is through the analysis of complete vessels. This is due to the fact that the stylistic features shared by both culturally close groups in this boundary area do not allow a clear distinction by observing only sherds.

\section{Data collection and model generation aimed to explain the Guaraní expansion}

In order to systematically analyze the great amount of information yielded by the Guaraní archaeological sites, a GIS database was created. The GIS platform offers the advantage of allowing the future incorporation of multiple data and variables (for example, landforms, phytogeography, palaeoenvironmental data or other archaeological traditions). The database was created with the purpose of broadening the interpretative potential of the available information. The sources of information used include scientific publications, reports, museum collections, lists of dates from radiocarbon laboratories, thematic maps, and three new ${ }^{14} \mathrm{C}$ dates (from Arroyo Malo, Ensenada del Bellaco, and Cementerio $\mathrm{A}^{\circ}$ Paicarabí y Fredes archaeological sites); therefore, its quality is considerably heterogeneous. Furthermore, some previous compilations of dates were used (Brochado, 1984; Noelli, 1999-2000; Rogge, 2005; Corrêa, 2009).

The database used for this study consists of an initial sample of 1177 archaeological sites (Table 1, Fig. 2). The sites must meet two general requirements: 1 ) that their material assemblages be clearly associated with a Guaraní settlement (findings of isolated Guaraní items were excluded), and 2) that they could be located in space (Guaraní sites with unclear geographic provenance data were excluded). While the sources present varying degrees of precision of site location, given the scale of the study area, the errors deriving from such disparity are of little relevance.

Within the initial sample, only 138 archaeological sites have numerical dates with its \pm error, obtained through the radiocarbon method ( ${ }^{14} \mathrm{C} ; n=145$ ) or thermoluminescence (TL; $n=103$ ). Between 1 and 33 dates per site were recorded, representing a total of 248 dates (Tables 2-5). In addition, five more sites were added which presented European materials (mainly glass beads)

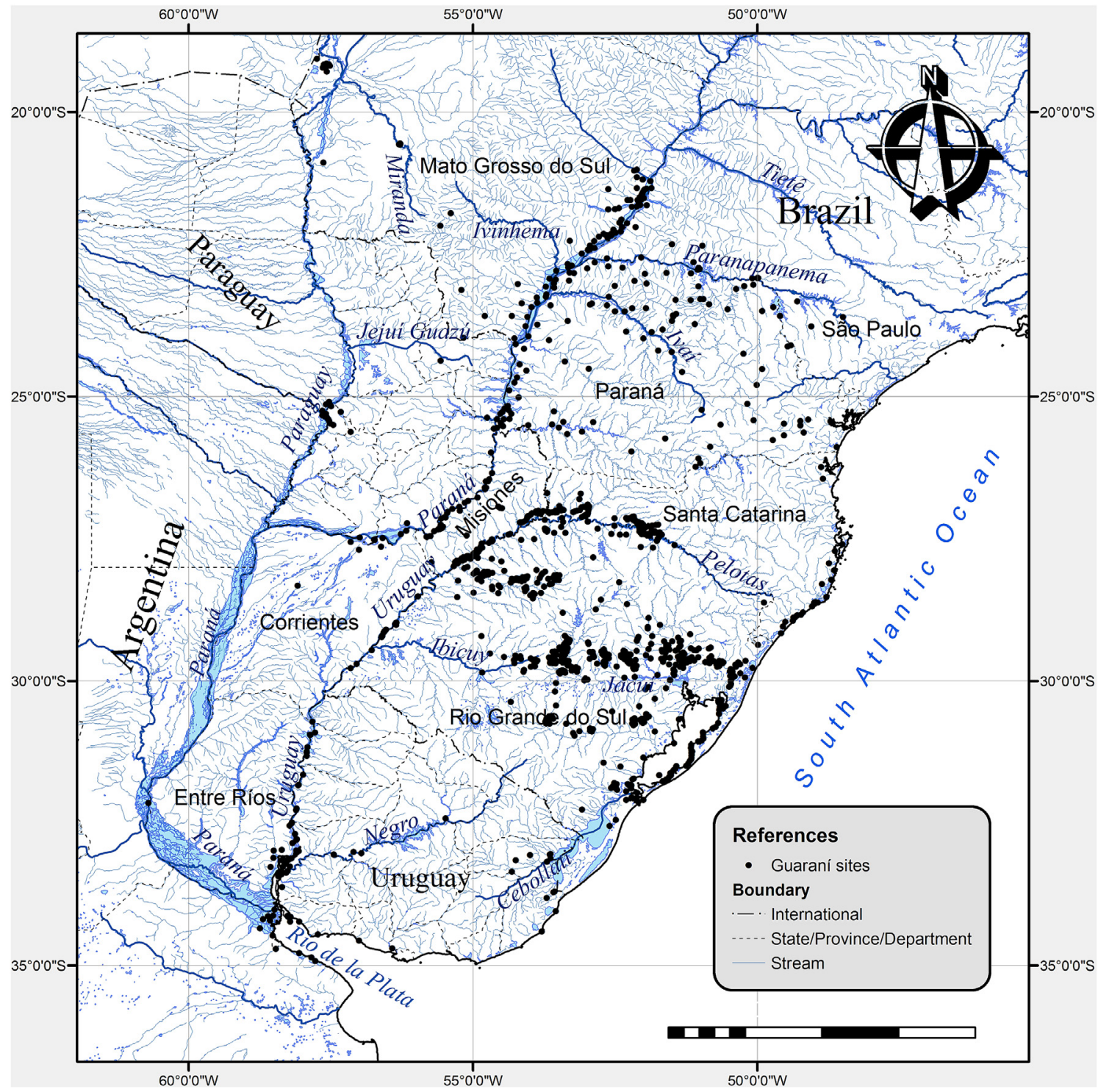

Fig. 2. Geographical distribution of the Guarani archaeological sites $(n=1177)$ in the La Plata Basin and adjacent areas. 
Table 1

Synthesis of Guaraní data used in the present study.

\begin{tabular}{|c|c|c|c|c|c|}
\hline \multicolumn{3}{|c|}{ Geographic location } & \multirow{2}{*}{$\frac{\text { Archaeological sites }}{12}$} & \multirow{2}{*}{$\frac{\text { Dated archaeological sites }}{1}$} & \multirow{2}{*}{$\frac{\text { Quantity of dates }}{1}$} \\
\hline La Plata Basin & & La Plata River & & & \\
\hline & \multirow[t]{5}{*}{ Uruguay River Basin } & Upper Uruguay & 266 & 15 & 17 \\
\hline & & Middle Uruguay & 22 & 0 & 0 \\
\hline & & Lower Uruguay & 30 & 3 & 5 \\
\hline & & Negro River Basin & 8 & 0 & 0 \\
\hline & & Ibicuí River Basin & 18 & 1 & 2 \\
\hline & \multirow[t]{6}{*}{ Paraná River Basin } & Upper Paraná & 203 & 48 & 122 \\
\hline & & Lower Paraná & 8 & 4 & 6 \\
\hline & & Batel marshes & 1 & 1 & 7 \\
\hline & & Ivaí River Basin & 32 & 11 & 16 \\
\hline & & Paranapanema River Basin & 55 & 11 & 15 \\
\hline & & Iguaçu River Basin & 22 & 4 & 5 \\
\hline & \multirow[t]{2}{*}{ Paraguay River Basin } & Miranda River Basin & 6 & 1 & 1 \\
\hline & & Paraguay River & 29 & 0 & 0 \\
\hline \multirow[t]{4}{*}{ Adjacent Areas } & & Guaíba Basin & 281 & 19 & 23 \\
\hline & & Camaquã River Basin & 43 & 1 & 1 \\
\hline & & Merín Lagoon and Cebollatí River Basin & 23 & 0 & 0 \\
\hline & & Atlantic Coast and Los Patos Lagoon & 118 & 18 & 27 \\
\hline Total & & & 1177 & 138 & 248 \\
\hline
\end{tabular}

assignable to the 16th and 17th centuries: Río Matanzas B (Rusconi, 1928), La Maza I (Ceruti and Crowder, 1973), Isla Vizcaíno (Araújo, 1900), Isla de Arriba (Diaz, 1977), and Isla Larga (Cabrera Pérez, 2011). Later, from a sample of these 143 sites, three successive models were created: a Digital Elevation Model (DEM), a Dispersion Routes Model (DRM), and a Temporal Visualization Model (TVM).

\subsection{Digital Elevation Model (DEM)}

For the DEM, a point cloud was delimited on the basis of the 143 recorded Guaraní sites. The $X$ and $Y$ variables were defined on the basis of the geographic position of the sites (latitude and longitude in the WGS 1984 coordinate system), and the $Z$ variable was defined using the median chronology expressed in calendar age of each sites' ${ }^{14} \mathrm{C}$ or TL date. Although the best indicator of sample chronology are the calibrated age ranges, the present model needs single values and the median age is one of the most stable estimates (Stuiver et al., 2013). The $X, Y$ and $Z$ tridimensional variables enabled the construction of an artificial topography (Fig. 3) where the height $(Z)$ represents time. In this elevation model, the higher points correspond to the sites with later dates. In the case of the sites with more than one date, each date was considered as an independent point (although the interpolation algorithm only used the older one). The radiocarbon dates were calibrated with the Calib 7.0.0 program (Stuiver et al., 2013), using 2 sigma and the curve for the Southern Hemisphere (SHCal13; Hogg et al., 2013). In the case of the thermoluminescence method, the dates do not require calibration, since laboratories report the date in calendar years before the analysis was carried out. Given the fact that for many sites this information is lacking, the dates by thermoluminescence were recalculated using the year when the age was published as reference. Although the present study incorporates dates obtained by different methods, significant biases between TL and ${ }^{14} \mathrm{C}$ ages were not observed.

On the basis of the point cloud, it was possible to determine a proper topography for hydrological analysis which could serve as a base for the next model. For this, it was necessary to avoid introducing a surface with exaggerated fracture lines or artificial depressions; and, at the same time, it was important not to under represent the points showing notable differences with neighboring points. Accordingly, spline interpolation was used for the DEM where the surface generated is soft and points intercepts, thus additionally keeping the number of artificial depressions considerably low (Mitas and Mitasova, 1999; Olaya Ferrero, 2004).

\subsection{Dispersion Routes Model (DRM)}

For the DRM, a drainage network was defined on the DEM topography by employing hydrographic analysis tools (ArcHydro Tools, ArcGIS 10.1; Maidment, 2002; Olivera et al., 2002; Metz et al., 2011). The construction of this drainage network is carried out on the basis of the flux direction in each cell comprising the DEM. This flux heads towards the lower adjacent cell (in our case, this is the one with the oldest dates). An eight-direction pour-point method (D8) hydrographic model was used in order to define which one is heading towards the water. In an ordinary hydrographic model, fluvial courses can be understood as cell assemblages through which a considerable amount of channeled water circulates, and basins as groups of such assemblages, whose flux ends up reaching a single cell (Olaya Ferrero, 2004). In the DRM, the dynamic of dispersion operates inversely to the flux direction in a real drainage network, in other words, the main stream, departing from the oldest point, drains into the smaller streams. Finally, on the basis of

Table 2

Dates from 0 to 300 AD. References: AR = Argentina; CRT = Corrientes; BR = Brazil; MS = Mato Grosso do Sul; PR = Paraná; RS = Rio Grande do Sul; SP = São Paulo.

\begin{tabular}{|c|c|c|c|c|c|c|c|c|c|}
\hline Site & $\begin{array}{l}\text { Identification } \\
\text { code }\end{array}$ & $\begin{array}{l}\text { Province or } \\
\text { state/country }\end{array}$ & Latitude & Longitude & Age years BP & $\begin{array}{l}\text { Age years } \\
\mathrm{AD}\end{array}$ & Method & Lab code & Reference \\
\hline Lagoa 1 & MS-PR-22 & MS/BR & -22.0969 & -52.3986 & $1800 \pm 40$ & 180 & $\mathrm{TL}$ & FATEC-185 & Kashimoto 1997 \\
\hline Acampamento Três & SP-BA-7 & $\mathrm{SP} / \mathrm{BR}$ & -23.5830 & -49.6000 & $1870 \pm 100$ & 190 & $\mathrm{C} 14$ & SI-418 & Chmyz 1967, Stuckenrath and Mielke 1970 \\
\hline João Batista & - & $\mathrm{PR} / \mathrm{BR}$ & -24.2140 & -51.5060 & $1700 \pm 230$ & 280 & $\mathrm{TL}$ & - & Carle and Da Silva 2007 in Corrêa 2009 \\
\hline Fazenda Dona Carlota 2 & PR-FI-140 & $\mathrm{PR} / \mathrm{BR}$ & -25.5200 & -54.4720 & $2010 \pm 75$ & 27 & C14 & SI-5028 & Chmyz 1983 in Noelli 1999-2000 \\
\hline Emilio Kieffer & RS-MJ-88 & $\mathrm{RS} / \mathrm{BR}$ & -29.6590 & -53.5550 & $1800 \pm 100$ & 274 & $\mathrm{C} 14$ & SI-2205 & Brochado 1971, 1984 \\
\hline San Miguel II & - & CRT/AR & -27.6890 & -57.0040 & $1860 \pm 50$ & 196 & $\mathrm{C} 14$ & LP-215 & Mujica $1995 a$ \\
\hline
\end{tabular}


Table 3

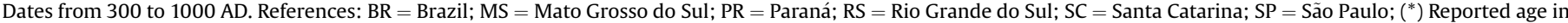
years AD.

\begin{tabular}{|c|c|c|c|c|c|c|c|c|c|}
\hline Site & $\begin{array}{l}\text { Identification } \\
\text { code }\end{array}$ & State/country & Latitude & Longitude & $\begin{array}{l}\text { Age years } \\
\text { BP }\end{array}$ & $\begin{array}{l}\text { Age years } \\
\text { AD }\end{array}$ & Method & Lab code & References \\
\hline Lagoa do Custodio 1 & MS-IV-08 & $\mathrm{MS} / \mathrm{BR}$ & -22.3844 & -52.8688 & $1170 \pm 140$ & 810 & $\mathrm{TL}$ & FATEC-164 & Martins et al. 1999 \\
\hline Lagoa do Custodio 1 & MS-IV-08 & MS/BR & -22.3844 & -52.8688 & $1200 \pm 150$ & 780 & $\mathrm{TL}$ & FATEC-148 & Kashimoto and Martins 2008 \\
\hline Ribeirão Taquari 1 & MS-PR-45 & MS/BR & -21.4170 & -52.0188 & $1380 \pm 40$ & 650 & $\mathrm{C} 14$ & Gif-12026 & Kashimoto and Martins 2008 \\
\hline Ilha Verde 2 & MS-PR-57 & MS/BR & -21.2090 & -51.8780 & $1000 \pm 100$ & 980 & $\mathrm{TL}$ & FATEC-253 & Kashimoto and Martins 2008 \\
\hline Ilha Verde 2 & MS-PR-57 & MS/BR & -21.2090 & -51.8780 & $1100 \pm 100$ & 880 & TL & FATEC-250 & Kashimoto and Martins 2008 \\
\hline Ilha Verde 2 & MS-PR-57 & $\mathrm{MS} / \mathrm{BR}$ & -21.2090 & -51.8780 & $1200 \pm 120$ & 780 & $\mathrm{TL}$ & FATEC-267 & Kashimoto and Martins 2008 \\
\hline Ilha Verde 2 & MS-PR-57 & $\mathrm{MS} / \mathrm{BR}$ & -21.2090 & -51.8780 & $1260 \pm 130$ & 720 & $\mathrm{TL}$ & FATEC-263 & Kashimoto and Martins 2008 \\
\hline Ilha Verde 2 & MS-PR-57 & $\mathrm{MS} / \mathrm{BR}$ & -21.2090 & -51.8780 & $1300 \pm 120$ & 680 & $\mathrm{TL}$ & FATEC-266 & Kashimoto and Martins 2008 \\
\hline Ilha Verde 2 & MS-PR-57 & MS/BR & -21.2090 & -51.8780 & $1400 \pm 120$ & 580 & $\mathrm{TL}$ & FATEC-262 & Kashimoto and Martins 2008 \\
\hline Ilha Verde 2 & MS-PR-57 & MS/BR & -21.2090 & -51.8780 & $1600 \pm 200$ & 380 & $\mathrm{TL}$ & FATEC-259 & Kashimoto and Martins 2008 \\
\hline Ilha Agua Limpa 1 & MS-PR-64 & MS/BR & -21.4400 & -52.0280 & $1248 \pm 100$ & 732 & $\mathrm{TL}$ & FATEC-194 & Kashimoto 1997 \\
\hline Alto Parana 14 & MS-PR-42 & MS/BR & -21.5110 & -51.9930 & $1110 \pm 110$ & 870 & $\mathrm{TL}$ & FATEC-398 & Kashimoto and Martins 2008 \\
\hline Río Pardo 7 & MS-PD-07 & MS/BR & -21.7070 & -52.6210 & $980 \pm 100$ & 1000 & $\mathrm{TL}$ & FATEC-402 & $\begin{array}{l}\text { Kashimoto 1997, Kashimoto and } \\
\text { Martins } 2008\end{array}$ \\
\hline Ragil & - & $\mathrm{SP} / \mathrm{BR}$ & -22.7380 & -51.0290 & $1660 \pm 170$ & 338 & $\mathrm{TL}$ & FATEC & Faccio 1998 \\
\hline Ragil II & - & $\mathrm{SP} / \mathrm{BR}$ & -22.7550 & -51.0110 & $1093 \pm 100$ & 887 & $\mathrm{TL}$ & FATEC & Faccio 1998 \\
\hline Porto Casanova 2 & SP-AS-14 & $\mathrm{SP} / \mathrm{BR}$ & -22.7670 & -51.0500 & $1130 \pm 150$ & 947 & C14 & SI-422 & $\begin{array}{l}\text { Chmyz 1967, Stuckenrath and Mielke } \\
1970\end{array}$ \\
\hline José Vieira & - & $\mathrm{SP} / \mathrm{BR}$ & -23.3630 & -52.9080 & $1380 \pm 150$ & 717 & C14 & Gsy 81 & Brochado 1984 \\
\hline SP-BA-7 & SP-BA-7 & $\mathrm{SP} / \mathrm{BR}$ & -23.5830 & -49.6000 & $1195 \pm 80$ & 886 & C14 & SI-1009 & $\begin{array}{l}\text { Chmyz 1967, Stuckenrath and Mielke } \\
1973\end{array}$ \\
\hline Rio Paranapanema & - & SP/BR & -24.0970 & -49.4100 & - & $820 \pm 150^{*}$ & $\mathrm{C} 14$ & - & Chmyz 1977 in Corrêa 2009 \\
\hline Restaurante Ivaí 2 & PR-FL-21 & $\mathrm{PR} / \mathrm{BR}$ & -23.5010 & -52.3330 & $1490 \pm 45$ & 610 & $\mathrm{C} 14$ & SI-1011 & $\begin{array}{l}\text { Stuckenrath and Mielke 1973, Brochado } \\
1984\end{array}$ \\
\hline João Batista & - & $\mathrm{PR} / \mathrm{BR}$ & -24.2140 & -51.5060 & $1519 \pm 200$ & 461 & $\mathrm{TL}$ & - & Carle and Da Silva 2007 in Corrêa 2009 \\
\hline Boreví 1 & PR-FI-99 & $\mathrm{PR} / \mathrm{BR}$ & -25.3960 & -54.4560 & $1565 \pm 70$ & 533 & $\mathrm{C} 14$ & SI-5019 & Chmyz 1983 in Noelli 1999-2000 \\
\hline Lagoa Seca & PR-FI-118 & $\mathrm{PR} / \mathrm{BR}$ & -25.4630 & -54.5090 & $1625 \pm 60$ & 479 & C14 & SI-5021 & Chmyz 1983 in Noelli 1999-2000 \\
\hline Taquapelagai & PR-FI-97 & $\mathrm{PR} / \mathrm{BR}$ & -25.5010 & -54.0300 & $1235 \pm 60$ & 565 & $\mathrm{C} 14$ & SI-5016 & Chmyz 1983 in Noelli 1999-2000 \\
\hline Fazenda Dona Carlota 4 & PR-FI-142 & $\mathrm{PR} / \mathrm{BR}$ & -25.5450 & -54.5740 & $1395 \pm 60$ & 686 & $\mathrm{C} 14$ & SI-5033 & Chmyz 1983 in Noelli 1999-2000 \\
\hline Aldeia da ZPE & - & $\mathrm{SC} / \mathrm{BR}$ & -28.2166 & -48.6967 & $1000 \pm 110$ & 980 & $\mathrm{TL}$ & - & Farias and Kneips, 2010 \\
\hline Aldeia da ZPE & - & $\mathrm{SC} / \mathrm{BR}$ & -28.2166 & -48.6967 & $1040 \pm 110$ & 940 & $\mathrm{TL}$ & - & Farias and Kneips, 2010 \\
\hline Aldeia da ZPE & - & $\mathrm{SC} / \mathrm{BR}$ & -28.2166 & -48.6967 & $1050 \pm 150$ & 930 & $\mathrm{TL}$ & - & Farias and Kneips, 2010 \\
\hline Linha Uruguay Sul 1 & RS-VZ-4 & $\mathrm{RS} / \mathrm{BR}$ & -27.8170 & -55.0500 & $1220 \pm 120$ & 859 & C14 & SI-708 & Stuckenrath and Mielke 1973 \\
\hline Albino Mazzari & RS-MJ-60 & $\mathrm{RS} / \mathrm{BR}$ & -29.4800 & -53.6000 & $1180 \pm 70$ & 906 & C14 & SI-2204 & $\begin{array}{l}\text { Brochado 1971,Schmitz and Brochado, } \\
1972\end{array}$ \\
\hline Albino Mazzari & RS-MJ-60 & $\mathrm{RS} / \mathrm{BR}$ & -29.4800 & -53.6000 & $1475 \pm 80$ & 610 & $\mathrm{C} 14$ & SI-2203 & Brochado 1971, 1984 \\
\hline Palmeira 2 & RS-S-282 & $\mathrm{RS} / \mathrm{BR}$ & -29.6670 & -50.9170 & $1380 \pm 110$ & 718 & C14 & SI-414 & $\begin{array}{l}\text { Miller 1967, Stuckenrath and Mielke } \\
1970\end{array}$ \\
\hline Capané 3 & RS-MJ-101 & $\mathrm{RS} / \mathrm{BR}$ & -29.8360 & -53.1700 & $1255 \pm 100$ & 826 & $\mathrm{C} 14$ & SI-2201 & $\begin{array}{l}\text { Brochado 1971, Schmitz and Brochado } \\
1972\end{array}$ \\
\hline RS-T-114 & RS-T-114 & $\mathrm{RS} / \mathrm{BR}$ & -29.2885 & -52.1195 & $1410 \pm 115$ & 570 & $\mathrm{TL}$ & LACIFID-USP & Kreutz 2008 \\
\hline
\end{tabular}

this DRM, a series of vectors were developed which indicates the possible directions of the people dispersion (Fig. 4).

\subsection{Temporal Visualization Model (TVM)}

Through the use of temporal analysis tools (Temporal Analyst, ArcGIS 10.1), a TVM was created to represent people expansion. This model was based on the gradual appearance of archaeological sites in a chronological order, and on the basis of their numerical dates in calendar years (Figs. 5-7). The sites are connected with polygons according to their geographic and temporal proximity. These polygons were traced following the direction of the vectors yielded by the DRM.

\section{Results and interpretation}

\subsection{Dispersion Routes Model (DRM)}

The DRM enabled the formulation of the possible dispersion routes used by the Guaraní in the past (Fig. 4). The first noticeable aspect about Fig. 4 is that the rivers were not included in the model as a variable. Once the obtained vectors are overlapped against a physical map of the study area they show an overall concurrence with the existent hydrographic network. This is due to the fact most of the Guaraní settlements were close to watercourses.

On the basis of the DRM, it was possible to delimit a potential early area in the Upper Paraná River, where new archaeological sites with early dates could be expected. From this area, we can identify seven main dispersion routes:

- Three major northern dispersion routes. Two of these routes are directed to the east and are associated with two of the main Paraná tributaries (Paranapanema and Ivaí rivers). The third route is directed to the west towards the Paraguay River, reaching its tributary, the Miranda River.

- Four major southern dispersion routes. A first route is directed to the southwest following the Paraná River and is limited to the north of the Argentinean province of Corrientes. Two routes have their axis in the Uruguay River, one going upstream to the east reaching the Pelotas River and the other continuing south to the La Plata River. The fourth route, which is originated in the Middle Uruguay River, flows east following the Ibicuí course to the watershed with the Jacuí River, and presents several ramifications heading north into the Brazilian state of Rio Grande do Sul and south into Uruguay. Through these ramifications, the Guaraní reached the Atlantic coast. 
Table 4

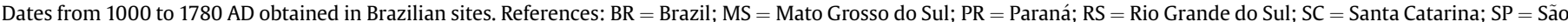

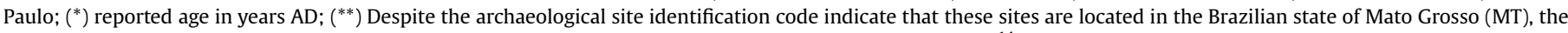

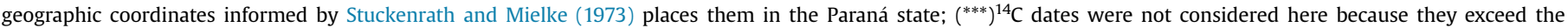
minimum-age limit of the method and are conventionally considered modern (Figini, 1993).

\begin{tabular}{|c|c|c|c|c|c|c|c|c|c|}
\hline Site & $\begin{array}{l}\text { Identification } \\
\text { code }\end{array}$ & $\begin{array}{l}\text { State/ } \\
\text { country }\end{array}$ & Latitude & Longitude & Age years BP & $\begin{array}{l}\text { Age years } \\
\mathrm{AD}\end{array}$ & Method & Lab code & Reference \\
\hline Lagoa 1 & MS-PR-22 & MS/BR & -22.0969 & -52.3986 & $370 \pm 20$ & 1562 & C14 & Gif-11073 & Kashimoto 1997 \\
\hline Lagoa do Custodio 1 & MS-IV-08 & MS/BR & -22.3844 & -52.8688 & $350 \pm 40$ & 1630 & $\mathrm{TL}$ & FATEC-136 & Kashimoto and Martins 2008 \\
\hline Lagoa do Custodio 1 & MS-IV-08 & $\mathrm{MS} / \mathrm{BR}$ & -22.3844 & -52.8688 & $375 \pm 45$ & 1605 & $\mathrm{TL}$ & FATEC-151 & Martins et al. 1999 \\
\hline Lagoa do Custodio 1 & MS-IV-08 & MS/BR & -22.3844 & -52.8688 & $435 \pm 50$ & 1545 & $\mathrm{TL}$ & FATEC-160 & Martins et al. 1999 \\
\hline Lagoa do Custodio 1 & MS-IV-08 & $\mathrm{MS} / \mathrm{BR}$ & -22.3844 & -52.8688 & $420 \pm 50$ & 1560 & $\mathrm{TL}$ & FATEC-157 & Martins et al. 1999 \\
\hline Lagoa do Custodio 1 & MS-IV-08 & MS/BR & -22.3844 & -52.8688 & $425 \pm 25$ & 1555 & $\mathrm{TL}$ & FATEC-183 & Martins et al. 1999 \\
\hline Lagoa do Custodio 1 & MS-IV-08 & MS/BR & -22.3844 & -52.8688 & $445 \pm 35$ & 1535 & $\mathrm{TL}$ & FATEC-140 & Martins et al. 1999 \\
\hline Lagoa do Custodio 1 & MS-IV-08 & $\mathrm{MS} / \mathrm{BR}$ & -22.3844 & -52.8688 & $460 \pm 55$ & 1520 & $\mathrm{TL}$ & FATEC-137 & Martins et al. 1999 \\
\hline Lagoa do Custodio 1 & MS-IV-08 & $\mathrm{MS} / \mathrm{BR}$ & -22.3844 & -52.8688 & $480 \pm 60$ & 1500 & $\mathrm{TL}$ & FATEC-141 & Martins et al. 1999 \\
\hline Lagoa do Custodio 1 & MS-IV-08 & MS/BR & -22.3844 & -52.8688 & $490 \pm 60$ & 1490 & $\mathrm{TL}$ & FATEC-144 & Martins et al. 1999 \\
\hline Lagoa do Custodio 1 & MS-IV-08 & $\mathrm{MS} / \mathrm{BR}$ & -22.3844 & -52.8688 & $500 \pm 60$ & 1480 & $\mathrm{TL}$ & FATEC-143 & Martins et al. 1999 \\
\hline Lagoa do Custodio 1 & MS-IV-08 & MS/BR & -22.3844 & -52.8688 & $505 \pm 60$ & 1475 & $\mathrm{TL}$ & FATEC-153 & Martins et al. 1999 \\
\hline Lagoa do Custodio 1 & MS-IV-08 & $\mathrm{MS} / \mathrm{BR}$ & -22.3844 & -52.8688 & $520 \pm 60$ & 1460 & $\mathrm{TL}$ & FATEC-159 & Kashimoto and Martins 2008 \\
\hline Lagoa do Custodio 1 & MS-IV-08 & MS/BR & -22.3844 & -52.8688 & $525 \pm 30$ & 1455 & $\mathrm{TL}$ & FATEC-165 & Martins et al. 1999 \\
\hline Lagoa do Custodio 1 & MS-IV-08 & $\mathrm{MS} / \mathrm{BR}$ & -22.3844 & -52.8688 & $545 \pm 65$ & 1435 & $\mathrm{TL}$ & FATEC-154 & Martins et al. 1999 \\
\hline Lagoa do Custodio 1 & MS-IV-08 & $\mathrm{MS} / \mathrm{BR}$ & -22.3844 & -52.8688 & $550 \pm 50$ & 1430 & $\mathrm{TL}$ & FATEC-90 & Martins et al. 1999 \\
\hline Lagoa do Custodio 1 & MS-IV-08 & MS/BR & -22.3844 & -52.8688 & $550 \pm 70$ & 1430 & $\mathrm{TL}$ & FATEC-138 & Martins et al. 1999 \\
\hline Lagoa do Custodio 1 & MS-IV-08 & $\mathrm{MS} / \mathrm{BR}$ & -22.3844 & -52.8688 & $590 \pm 70$ & 1390 & $\mathrm{TL}$ & FATEC-161 & Martins et al. 1999 \\
\hline Lagoa do Custodio 1 & MS-IV-08 & $\mathrm{MS} / \mathrm{BR}$ & -22.3844 & -52.8688 & $595 \pm 70$ & 1385 & $\mathrm{TL}$ & FATEC-145 & Martins et al. 1999 \\
\hline Lagoa do Custodio 1 & MS-IV-08 & MS/BR & -22.3844 & -52.8688 & $600 \pm 80$ & 1380 & $\mathrm{TL}$ & FATEC-142 & Martins et al. 1999 \\
\hline Lagoa do Custodio 1 & MS-IV-08 & MS/BR & -22.3844 & -52.8688 & $605 \pm 70$ & 1375 & $\mathrm{TL}$ & FATEC-158 & Martins et al. 1999 \\
\hline Lagoa do Custodio 1 & MS-IV-08 & $\mathrm{MS} / \mathrm{BR}$ & -22.3844 & -52.8688 & $610 \pm 75$ & 1370 & $\mathrm{TL}$ & FATEC-152 & Martins et al. 1999 \\
\hline Lagoa do Custodio 1 & MS-IV-08 & MS/BR & -22.3844 & -52.8688 & $625 \pm 50$ & 1355 & $\mathrm{TL}$ & FATEC-146 & Martins et al. 1999 \\
\hline Lagoa do Custodio 1 & MS-IV-08 & $\mathrm{MS} / \mathrm{BR}$ & -22.3844 & -52.8688 & $680 \pm 80$ & 1300 & $\mathrm{TL}$ & FATEC-149 & Martins et al. 1999 \\
\hline Lagoa do Custodio 1 & MS-IV-08 & MS/BR & -22.3844 & -52.8688 & $705 \pm 17$ & 1275 & $\mathrm{TL}$ & FATEC-LVD1259 & Martins et al. 1999 \\
\hline Lagoa do Custodio 1 & MS-IV-08 & $\mathrm{MS} / \mathrm{BR}$ & -22.3844 & -52.8688 & $750 \pm 80$ & 1230 & $\mathrm{TL}$ & FATEC-89 & Martins et al. 1999 \\
\hline Lagoa do Custodio 1 & MS-IV-08 & $\mathrm{MS} / \mathrm{BR}$ & -22.3844 & -52.8688 & $795 \pm 10$ & 1185 & $\mathrm{TL}$ & FATEC-156 & Martins et al. 1999 \\
\hline Lagoa do Custodio 1 & MS-IV-08 & MS/BR & -22.3844 & -52.8688 & $795 \pm 95$ & 1185 & $\mathrm{TL}$ & FATEC-150 & Martins et al. 1999 \\
\hline Lagoa do Custodio 1 & MS-IV-08 & MS/BR & -22.3844 & -52.8688 & $800 \pm 100$ & 1180 & $\mathrm{TL}$ & FATEC-139 & Martins et al. 1999 \\
\hline Lagoa do Custodio 1 & MS-IV-08 & MS/BR & -22.3844 & -52.8688 & $835 \pm 90$ & 1145 & $\mathrm{TL}$ & FATEC-162 & Martins et al. 1999 \\
\hline Lagoa do Custodio 1 & MS-IV-08 & $\mathrm{MS} / \mathrm{BR}$ & -22.3844 & -52.8688 & $930 \pm 110$ & 1050 & $\mathrm{TL}$ & FATEC-166 & Martins et al. 1999 \\
\hline Lagoa do Custodio 1 & MS-IV/08 & $\mathrm{MS} / \mathrm{BR}$ & -22.3844 & -52.8688 & $950 \pm 115$ & 1030 & $\mathrm{TL}$ & FATEC-163 & Kashimoto and Martins 2008 \\
\hline Quiterói 1 & MS-PR-08 & MS/BR & -22.0969 & -52.3986 & $320 \pm 35$ & 1660 & $\mathrm{TL}$ & FATEC-94 & Martins et al. 1999 \\
\hline Quiterói 5 & - & $\mathrm{MS} / \mathrm{BR}$ & -22.1593 & -52.6606 & $610 \pm 60$ & 1370 & $\mathrm{TL}$ & FATEC-118 & Martins et al. 1999 \\
\hline Río Taquari 4 & MS-PR-48 & $\mathrm{MS} / \mathrm{BR}$ & -21.4170 & -52.0188 & $380 \pm 40$ & 1600 & $\mathrm{TL}$ & FATEC-108 & $\begin{array}{l}\text { Kashimoto } 1997 \text {, Kashimoto } \\
\text { and Martins } 2008\end{array}$ \\
\hline Río Taquari 1 & MS-PR-45 & MS/BR & -21.4170 & -52.0188 & $410 \pm 40$ & 1570 & $\mathrm{TL}$ & FATEC-107 & $\begin{array}{l}\text { Kashimoto 1997, Kashimoto } \\
\text { and Martins } 2008\end{array}$ \\
\hline Cabeceira Perdida 1 & MS-PR-55 & MS/BR & -21.2396 & -51.9711 & $565 \pm 32$ & 1415 & $\mathrm{TL}$ & FATEC & Kashimoto 1997 \\
\hline Maracaju 1 & MS-MJ-1 & $\mathrm{MS} / \mathrm{BR}$ & -21.7743 & -55.3896 & $610 \pm 50$ & 1349 & $\mathrm{C} 14$ & Gif-8330 & Kashimoto and Martins, 2008 \\
\hline Maracaju 1 & MS-MJ-1 & $\mathrm{MS} / \mathrm{BR}$ & -21.7743 & -55.3896 & $830 \pm 80$ & 1150 & $\mathrm{TL}$ & Lab Fisica USP & Kashimoto and Martins, 2008 \\
\hline Córrego Lalima & MS-MI-01 & MS/BR & -20.5678 & -56.2823 & $970 \pm 60$ & 1111 & C14 & Beta-238765 & Bespalez 2010 \\
\hline Ilha Água Limpa 1 & MS-PR-64 & $\mathrm{MS} / \mathrm{BR}$ & -20.7790 & -51.6890 & $1015 \pm 75$ & 1085 & $\mathrm{C} 14$ & Gif-10039 & Kashimoto 1997 \\
\hline Ilha Verde 2 & MS-PR-57 & MS/BR & -21.0260 & -52.1640 & $380 \pm 40$ & 1600 & $\mathrm{TL}$ & FATEC-264 & Kashimoto and Martins 2008 \\
\hline Ilha Verde 2 & MS-PR-57 & $\mathrm{MS} / \mathrm{BR}$ & -21.2090 & -51.8780 & $510 \pm 50$ & 1438 & $\mathrm{C} 14$ & Beta-218207 & Kashimoto and Martins 2008 \\
\hline Ilha Verde 2 & MS-PR-57 & $\mathrm{MS} / \mathrm{BR}$ & -21.2090 & -51.8780 & $520 \pm 50$ & 1460 & $\mathrm{TL}$ & FATEC-261 & Kashimoto and Martins 2008 \\
\hline Ilha Verde 2 & MS-PR-57 & MS/BR & -21.2090 & -51.8780 & $630 \pm 60$ & 1350 & $\mathrm{TL}$ & FATEC-256 & Kashimoto and Martins 2008 \\
\hline Ilha Verde 2 & MS-PR-57 & $\mathrm{MS} / \mathrm{BR}$ & -21.2090 & -51.8780 & $700 \pm 70$ & 1280 & $\mathrm{TL}$ & FATEC-265 & Kashimoto and Martins 2008 \\
\hline Ilha Verde 2 & MS-PR-57 & $\mathrm{MS} / \mathrm{BR}$ & -21.2090 & -51.8780 & $880 \pm 80$ & 1100 & $\mathrm{TL}$ & FATEC-255 & Kashimoto and Martins 2008 \\
\hline Ilha Verde 2 & MS-PR-57 & MS/BR & -21.2090 & -51.8780 & $900 \pm 90$ & 1080 & $\mathrm{TL}$ & FATEC-251 & Kashimoto and Martins 2008 \\
\hline Río Taquari 2 & MS-PR-46 & $\mathrm{MS} / \mathrm{BR}$ & -21.2090 & -51.8780 & $280 \pm 15$ & 1700 & $\mathrm{TL}$ & FATEC-192 & $\begin{array}{l}\text { Kashimoto 1997, Kashimoto } \\
\text { and Martins } 2008\end{array}$ \\
\hline Córrego Azul 1 & MS-PR-41 & $\mathrm{MS} / \mathrm{BR}$ & -21.4280 & -52.0180 & $245 \pm 15$ & 1735 & $\mathrm{TL}$ & FATEC-191 & $\begin{array}{l}\text { Kashimoto 1997, Kashimoto } \\
\text { and Martins } 2008\end{array}$ \\
\hline Alto Paraná 14 & MS-PR-42 & MS/BR & -21.4920 & -52.0680 & $570 \pm 60$ & 1410 & $\mathrm{TL}$ & FATEC-397 & Kashimoto and Martins 2008 \\
\hline Alto Paraná 14 & MS-PR-42 & MS/BR & -21.5110 & -51.9930 & $840 \pm 40$ & 1238 & C14 & Gif-11226 & Kashimoto and Martins 2008 \\
\hline Alto Paraná 12 & MS-PR-39 & $\mathrm{MS} / \mathrm{BR}$ & -21.5110 & -51.9930 & $580 \pm 40$ & 1400 & $\mathrm{TL}$ & FATEC-190 & Kashimoto 1997 \\
\hline Alto Paraná 8 & MS-PR-35 & $\mathrm{MS} / \mathrm{BR}$ & -21.5410 & -52.1060 & $390 \pm 40$ & 1590 & $\mathrm{TL}$ & FATEC-98 & $\begin{array}{l}\text { Kashimoto 1997, Kashimoto } \\
\text { and Martins } 2008\end{array}$ \\
\hline Alto Paraná 8 & MS-PR-35 & MS/BR & -21.6310 & -52.0580 & $625 \pm 40$ & 1355 & $\mathrm{TL}$ & FATEC-189 & $\begin{array}{l}\text { Kashimoto 1997, Kashimoto } \\
\text { and Martins } 2008\end{array}$ \\
\hline Cabeceira Perdida 1 & MS-PR-55 & MS/BR & -21.6310 & -52.0580 & $565 \pm 32$ & 1415 & $\mathrm{TL}$ & FATEC-193 & Kashimoto 1997 \\
\hline Córrego Uerê & MS-PD-06 & $\mathrm{MS} / \mathrm{BR}$ & -21.7030 & -52.4310 & $415 \pm 40$ & 1474 & $\mathrm{TL}$ & FATEC-406 & Noelli 1999-2000 \\
\hline Córrego Uerê & MS-PD-06 & $\mathrm{MS} / \mathrm{BR}$ & -21.7030 & -52.4310 & $240 \pm 30$ & 1752 & $\mathrm{C} 14$ & Gif-10038 & $\begin{array}{l}\text { Kashimoto 1997, Kashimoto } \\
\text { and Martins } 2008\end{array}$ \\
\hline Córrego Uerê & MS-PD-06 & MS/BR & -21.7030 & -52.4310 & $250 \pm 25$ & 1730 & $\mathrm{TL}$ & FATEC-405 & $\begin{array}{l}\text { Kashimoto 1997, Kashimoto } \\
\text { and Martins } 2008\end{array}$ \\
\hline Río Pardo 7 & MS-PD-07 & MS/BR & -21.7030 & -52.5000 & $275 \pm 20$ & 1705 & $\mathrm{TL}$ & FATEC-188 & $\begin{array}{l}\text { Kashimoto 1997, Kashimoto } \\
\text { and Martins } 2008\end{array}$ \\
\hline
\end{tabular}


Table 4 (continued)

\begin{tabular}{|c|c|c|c|c|c|c|c|c|c|}
\hline Site & $\begin{array}{l}\text { Identification } \\
\text { code }\end{array}$ & $\begin{array}{l}\text { State/ } \\
\text { country }\end{array}$ & Latitude & Longitude & Age years BP & $\begin{array}{l}\text { Age years } \\
\text { AD }\end{array}$ & Method & Lab code & Reference \\
\hline Río Pardo 7 & MS-PD-07 & MS/BR & -21.7070 & -52.6210 & $610 \pm 60$ & 1370 & $\mathrm{TL}$ & FATEC-399 & $\begin{array}{l}\text { Kashimoto 1997, Kashimoto } \\
\text { and Martins } 2008\end{array}$ \\
\hline Río Pardo 7 & MS-PD-07 & MS/BR & -21.7070 & -52.6210 & $750 \pm 75$ & 1230 & $\mathrm{TL}$ & FATEC-400 & $\begin{array}{l}\text { Kashimoto } 1997, \text { Kashimoto } \\
\text { and Martins } 2008\end{array}$ \\
\hline Córrego Caraguatá 2 & MS-PR-28 & MS/BR & -21.9400 & -52.4210 & $350 \pm 35$ & 1630 & $\mathrm{TL}$ & FATEC-116 & Kashimoto and Martins 2008 \\
\hline Córrego S. Lourenço 1 & MS-PR-26 & MS/BR & -22.0180 & -52.3770 & $290 \pm 30$ & 1690 & $\mathrm{TL}$ & FATEC-123 & Kashimoto and Martins 2008 \\
\hline Córrego S. Lourenço 1 & MS-PR-26 & MS/BR & -22.0180 & -52.3770 & $380 \pm 40$ & 1600 & $\mathrm{TL}$ & FATEC-122 & Kashimoto and Martins 2008 \\
\hline Lagoa Ariranha 1 & MS-PR-22 & MS/BR & -22.0970 & -52.3990 & $370 \pm 20$ & 1610 & $\mathrm{TL}$ & FATEC-185 & Kashimoto and Martins 2008 \\
\hline $\begin{array}{l}\text { Ribeirão Quiteroizinho } \\
\quad 1\end{array}$ & MS-PR-18 & MS/BR & -22.1130 & -52.4930 & $380 \pm 40$ & 1600 & $\mathrm{TL}$ & FATEC-106 & Kashimoto and Martins 2008 \\
\hline Ribeirão Quiterói 6 & MS-PR-13 & MS/BR & -22.1360 & -52.7030 & $239 \pm 10$ & 1741 & $\mathrm{TL}$ & FATEC-184 & Kashimoto 1997, \\
\hline Río Pardo 4 & MS-PD-04 & MS/BR & -22.1860 & -52.7850 & $432 \pm 32$ & 1548 & $\mathrm{TL}$ & FATEC-187 & Kashimoto 1997 \\
\hline Ribeirão Quiterói 1 & MS-PR-08 & MS/BR & -22.2070 & -52.6280 & $300 \pm 50$ & 1680 & $\mathrm{TL}$ & FATEC-95 & Kashimoto and Martins 2008 \\
\hline Ribeirão Quiterói 1 & MS-PR-08 & MS/BR & -22.2070 & -52.6280 & $320 \pm 35$ & 1660 & $\mathrm{TL}$ & FATEC-94 & Kashimoto and Martins 2008 \\
\hline Ribeirão Quiterói 1 & MS-PR-08 & MS/BR & -22.2070 & -52.6280 & $350 \pm 30$ & 1630 & $\mathrm{TL}$ & FATEC-96 & Kashimoto and Martins 2008 \\
\hline Baia da Onça 1 & - & MS/BR & -22.3980 & -52.9250 & $540 \pm 60$ & 1440 & $\mathrm{TL}$ & FATEC-117 & Kashimoto and Martins 2008 \\
\hline Rio Ivinhema 1 & MS-IV-09 & MS/BR & -23.2440 & -53.7150 & $570 \pm 40$ & 1410 & $\mathrm{TL}$ & FATEC-996 & Kashimoto and Martins 2008 \\
\hline Rio Ivinhema 1 & MS-IV-09 & MS/BR & -23.2440 & -53.7150 & $600 \pm 57$ & 1380 & $\mathrm{TL}$ & FATEC-997 & Kashimoto and Martins 2008 \\
\hline Alto Paraná 46 & MS-PR-98 & MS/BR & -23.5970 & -54.0420 & $480 \pm 30$ & 1500 & $\mathrm{TL}$ & FATEC-196 & $\begin{array}{l}\text { Kashimoto 1997, Kashimoto } \\
\text { and Martins } 2008\end{array}$ \\
\hline Rio Amambai 1 & - & MS/BR & -23.9640 & -55.1640 & $540 \pm 40$ & 1440 & $\mathrm{TL}$ & FATEC-127 & Kashimoto and Martins 2008 \\
\hline Alvim & - & $\mathrm{SP} / \mathrm{BR}$ & -22.3190 & -51.4990 & $906 \pm 90$ & 1074 & $\mathrm{TL}$ & - & Faccio 1992 \\
\hline Alvim & - & $\mathrm{SP} / \mathrm{BR}$ & -22.3190 & -51.4990 & $978 \pm 100$ & 1014 & $\mathrm{TL}$ & - & Faccio 1992 \\
\hline Neves & - & $\mathrm{SP} / \mathrm{BR}$ & -22.3480 & -50.9670 & $755 \pm 80$ & 1225 & $\mathrm{TL}$ & FATEC & Faccio 1998 \\
\hline Porto Casanova & - & $\mathrm{SP} / \mathrm{BR}$ & -22.6340 & -51.1210 & $980 \pm 100$ & 1104 & $\mathrm{C} 14$ & - & Chmyz 1977, in Corrêa 2009 \\
\hline Porto Casanova 2 & SP-AS-14 & $\mathrm{SP} / \mathrm{BR}$ & -22.8330 & -51.1670 & $908 \pm 100$ & 1162 & $\mathrm{C} 14$ & SI-709 & Stuckenrath and Mielke 1973 \\
\hline SP-BA-7 & SP-BA-7 & $\mathrm{SP} / \mathrm{BR}$ & -23.5830 & -49.6000 & $850 \pm 150$ & 1200 & $\mathrm{C} 14$ & SI-417 & $\begin{array}{l}\text { Chmyz } 1967 . \text { Stuckenrath and } \\
\text { Mielke } 1970\end{array}$ \\
\hline Rio Paranapanema & - & $\mathrm{SP} / \mathrm{BR}$ & -24.0970 & -49.4100 & - & $1190 \pm 50^{*}$ & $\mathrm{C} 14$ & - & Chmyz 1977 in Corrêa 2009 \\
\hline $\begin{array}{l}\text { Pesqueiro Barragem de } \\
\text { Rosana }\end{array}$ & PR-NL-7 & $\mathrm{PR} / \mathrm{BR}$ & -22.6410 & -52.8560 & $530 \pm 55$ & 1429 & $\mathrm{C} 14$ & SI-6400 & Chmyz and Chmyz 1986 \\
\hline MT-IV-1 & MT-IV-1 & $\mathrm{PR} / \mathrm{BR}^{* *}$ & -23.5000 & -52.5000 & $180 \pm 60$ & Modern*** & $\mathrm{C} 14$ & SI-1018 & $\begin{array}{l}\text { Chmyz 1969a, Stuckenrath and } \\
\text { Mielke } 1973\end{array}$ \\
\hline MT-IV-1 & MT-IV-1 & $\mathrm{PR} / \mathrm{BR}^{* *}$ & -23.5000 & -52.5000 & $260 \pm 70$ & 1709 & $\mathrm{C} 14$ & SI-1016 & $\begin{array}{l}\text { Chmyz } 1969 a \text {, Stuckenrath and } \\
\text { Mielke } 1973\end{array}$ \\
\hline MT-IV-1 & MT-IV-1 & $\mathrm{PR} / \mathrm{BR}^{* *}$ & -23.5000 & -52.5000 & $475 \pm 45$ & 1458 & $\mathrm{C} 14$ & SI-1017 & $\begin{array}{l}\text { Chmyz 1969a, Stuckenrath and } \\
\text { Mielke } 1973\end{array}$ \\
\hline MT-IV-2 & MT-IV-2 & $\mathrm{PR} / \mathrm{BR}^{* *}$ & -23.6670 & -53.3330 & $110 \pm 60$ & Modern*** & $\mathrm{C} 14$ & SI-1019 & $\begin{array}{l}\text { Chmyz 1969a, Stuckenrath and } \\
\text { Mielke } 1973\end{array}$ \\
\hline Corredeira dos Caiuá & PR-JA-1 & $\mathrm{PR} / \mathrm{BR}$ & -22.9170 & -50.0000 & $760 \pm 50$ & 1288 & $\mathrm{C} 14$ & SI-140 & Long 1965 \\
\hline Rio Condor 3 & PR-FL-13 & $\mathrm{PR} / \mathrm{BR}$ & -23.5000 & -52.5000 & $135 \pm 120$ & Modern*** & C14 & SI-698 & $\begin{array}{l}\text { Chmyz 1969a, Stuckenrath and } \\
\text { Mielke } 1973\end{array}$ \\
\hline Rancho Leôncio 2 & PR-FL-5 & $\mathrm{PR} / \mathrm{BR}$ & -23.5000 & -52.5000 & $300 \pm 115$ & 1649 & $\mathrm{C} 14$ & SI-693 & $\begin{array}{l}\text { Chmyz 1969a, Stuckenrath and } \\
\text { Mielke } 1973\end{array}$ \\
\hline Rancho Leôncio 2 & PR-FL-5 & $\mathrm{PR} / \mathrm{BR}$ & -23.5000 & -52.5000 & $470 \pm 100$ & 1493 & $\mathrm{C} 14$ & SI-694 & $\begin{array}{l}\text { Chmyz 1969a, Stuckenrath and } \\
\text { Mielke } 1973\end{array}$ \\
\hline Segunda Corredeira & PR-FL-23 & $\mathrm{PR} / \mathrm{BR}$ & -23.5000 & -52.3330 & $560 \pm 30$ & 1418 & $\mathrm{C} 14$ & SI-700 & $\begin{array}{l}\text { Stuckenrath and Mielke 1973, } \\
\text { Brochado } 1984\end{array}$ \\
\hline Rio Condor 5 & PR-FL-15 & $\mathrm{PR} / \mathrm{BR}$ & -23.5000 & -52.5000 & $590 \pm 70$ & 1393 & $\mathrm{C} 14$ & SI-699 & $\begin{array}{l}\text { Brochado } 1973 \text {, Stuckenrath } \\
\text { and Mielke } 1973\end{array}$ \\
\hline Sitio Geraldo & PR-ST-1 & $\mathrm{PR} / \mathrm{BR}$ & -23.5000 & -52.5000 & $610 \pm 120$ & 1379 & $\mathrm{C} 14$ & SI-696 & $\begin{array}{l}\text { Brochado } 1973, \text { Stuckenrath } \\
\text { and Mielke } 1973\end{array}$ \\
\hline Sitio Geraldo & PR-ST-1 & $\mathrm{PR} / \mathrm{BR}$ & -23.5000 & -52.5000 & $1065 \pm 95$ & 1026 & $\mathrm{C} 14$ & SI-695 & $\begin{array}{l}\text { Brochado } 1973, \text { Stuckenrath } \\
\text { and Mielke } 1973\end{array}$ \\
\hline Porto de Areia 2 & PR-QN-2 & PR/BR & -23.5010 & -52.5000 & $540 \pm 60$ & 1424 & C14 & SI-697 & $\begin{array}{l}\text { Chmyz 1969a, Stuckenrath and } \\
\text { Mielke } 1973\end{array}$ \\
\hline Sete Quedas 3 & PR-FO-4 & $\mathrm{PR} / \mathrm{BR}$ & -24.0770 & -54.2470 & $760 \pm 40$ & 1287 & $\mathrm{C} 14$ & SI-5039 & $\begin{array}{l}\text { Chmyz } 1983 \text { in Noelli } 1999 \\
-2000\end{array}$ \\
\hline Porto Estamata & PR-FI-98 & $\mathrm{PR} / \mathrm{BR}$ & -25.1720 & -54.3880 & $190 \pm 75$ & Modern*** & $\mathrm{C} 14$ & SI-5018 & $\begin{array}{l}\text { Chmyz } 1983 \text { in Noelli } 1999 \\
-2000\end{array}$ \\
\hline Paineira & PR-FI-103 & $\mathrm{PR} / \mathrm{BR}$ & -25.2080 & -54.4420 & $600 \pm 60$ & 1388 & $\mathrm{C} 14$ & SI-5029 & $\begin{array}{l}\text { Chmyz } 1983 \text { in Noelli } 1999 \\
-2000\end{array}$ \\
\hline Cafezal 2 & PR-FI-127 & $\mathrm{PR} / \mathrm{BR}$ & -25.2380 & -54.3690 & $590 \pm 55$ & 1397 & $\mathrm{C} 14$ & SI-5024 & $\begin{array}{l}\text { Chmyz } 1983 \text { in Noelli } 1999 \\
-2000\end{array}$ \\
\hline Borevi 2 & PR-FI-100 & $\mathrm{PR} / \mathrm{BR}$ & -25.2690 & -54.4780 & $625 \pm 55$ & 1358 & $\mathrm{C} 14$ & SI-5020 & $\begin{array}{l}\text { Chmyz } 1983 \text { in Noelli } 1999 \\
-2000\end{array}$ \\
\hline Fazenda Dona Carlota 1 & PR-FI-104 & $\mathrm{PR} / \mathrm{BR}$ & -25.2990 & -54.3250 & $415 \pm 75$ & 1536 & $\mathrm{C} 14$ & SI-5032 & $\begin{array}{l}\text { Chmyz } 1983 \text { in Noelli } 1999 \\
-2000\end{array}$ \\
\hline Foz do Bela Vista 1 & PR-FI-22 & PR/BR & -25.3900 & -54.3560 & $230 \pm 80$ & 1751 & C14 & SI-5015 & $\begin{array}{l}\text { Chmyz } 1983 \text { in Noelli } 1999 \\
-2000\end{array}$ \\
\hline Rio Dois Irmãos & PR-FI-112 & $\mathrm{PR} / \mathrm{BR}$ & -25.3970 & -54.5780 & $700 \pm 55$ & 1338 & $\mathrm{C} 14$ & SI-5036 & $\begin{array}{l}\text { Chmyz } 1983 \text { in Noelli } 1999 \\
-2000\end{array}$ \\
\hline
\end{tabular}


Table 4 (continued)

\begin{tabular}{|c|c|c|c|c|c|c|c|c|c|}
\hline Site & $\begin{array}{l}\text { Identification } \\
\text { code }\end{array}$ & $\begin{array}{l}\text { State/ } \\
\text { country }\end{array}$ & Latitude & Longitude & Age years BP & $\begin{array}{l}\text { Age years } \\
\mathrm{AD}\end{array}$ & Method & Lab code & Reference \\
\hline Lagoa Seca & PR-FI-118 & $\mathrm{PR} / \mathrm{BR}$ & -25.4630 & -54.5090 & $205 \pm 80$ & 1771 & $\mathrm{C} 14$ & SI-5022 & $\begin{array}{l}\text { Chmyz } 1983 \text { in Noelli } 1999 \\
-2000\end{array}$ \\
\hline Lagoa Seca & PR-FI-118 & $\mathrm{PR} / \mathrm{BR}$ & -25.4630 & -54.5090 & $340 \pm 60$ & 1569 & C14 & SI-5023 & $\begin{array}{l}\text { Chmyz } 1983 \text { in Noelli } 1999 \\
-2000\end{array}$ \\
\hline Rio Ferraria 1 & PR-CT-54 & $\mathrm{PR} / \mathrm{BR}$ & -25.4760 & -49.5300 & $528 \pm 70$ & 1432 & $\mathrm{C} 14$ & Beta-22645 & $\begin{array}{l}\text { Chmyz } 1983 \text { in Noelli } 1999 \\
-2000\end{array}$ \\
\hline Taquapelagai & PR-FI-97 & $\mathrm{PR} / \mathrm{BR}$ & -25.5010 & -54.0300 & $255 \pm 80$ & 1723 & $\mathrm{C} 14$ & SI-5017 & $\begin{array}{l}\text { Chmyz } 1983 \text { in Noelli } 1999 \\
-2000\end{array}$ \\
\hline Fazenda Dona Carlota 2 & PR-FI-140 & $\mathrm{PR} / \mathrm{BR}$ & -25.5200 & -54.4720 & $745 \pm 75$ & 1302 & C14 & SI-5027 & $\begin{array}{l}\text { Chmyz } 1983 \text { in Noelli } 1999 \\
-2000\end{array}$ \\
\hline Fazenda Dona Carlota 4 & PR-FI-142 & $\mathrm{PR} / \mathrm{BR}$ & -25.5450 & -54.5740 & $395 \pm 60$ & 1546 & $\mathrm{C} 14$ & SI-5034 & $\begin{array}{l}\text { Chmyz } 1983 \text { in Noelli } 1999 \\
-2000\end{array}$ \\
\hline Sete Quedas 2 & PR-FO-3 & $\mathrm{PR} / \mathrm{BR}$ & -26.0840 & -51.0340 & $490 \pm 60$ & 1455 & C14 & SI-5040 & $\begin{array}{l}\text { Chmyz } 1983 \text { in Noelli } 1999 \\
-2000\end{array}$ \\
\hline Elevatório de Águas & PR-UV-16 & $\mathrm{PR} / \mathrm{BR}$ & -26.1670 & -51.0000 & $500 \pm 45$ & 1442 & C14 & SI-1015 & $\begin{array}{l}\text { Chmyz 1969b, Stuckenrath and } \\
\text { Mielke } 1973\end{array}$ \\
\hline João A. Guarantier & SC-U-54 & $\mathrm{SC} / \mathrm{BR}$ & -27.0830 & -53.0330 & $250 \pm 90$ & 1727 & C14 & SI-546 & $\begin{array}{l}\text { Piazza 1969, Stuckenrath and } \\
\text { Mielke } 1972\end{array}$ \\
\hline São Carlos & SC-VX-5 & $\mathrm{SC} / \mathrm{BR}$ & -27.1000 & -53.0170 & $490 \pm 70$ & 1461 & C14 & SI-548 & $\begin{array}{l}\text { Piazza 1969, Stuckenrath and } \\
\text { Mielke } 1972\end{array}$ \\
\hline Caxambu do Sul 1 & SC-U-55 & $\mathrm{SC} / \mathrm{BR}$ & -27.1170 & -53.0830 & $510 \pm 70$ & 1444 & $\mathrm{C} 14$ & SI-547 & $\begin{array}{l}\text { Piazza 1969, Stuckenrath and } \\
\text { Mielke } 1972\end{array}$ \\
\hline Caxambu do Sul 1 & SC-U-55 & $\mathrm{SC} / \mathrm{BR}$ & -27.1170 & -53.0830 & $620 \pm 80$ & 1365 & C14 & SI-550 & $\begin{array}{l}\text { Piazza 1969, Stuckenrath and } \\
\text { Mielke } 1972\end{array}$ \\
\hline Barra Escondida I & SC-U-69 & $\mathrm{SC} / \mathrm{BR}$ & -27.1330 & -53.4330 & $1070 \pm 100$ & 1019 & $\mathrm{C} 14$ & SI-549 & $\begin{array}{l}\text { Piazza 1969, Stuckenrath and } \\
\text { Mielke } 1972\end{array}$ \\
\hline Mondaí 1 & SC-U-53 & $\mathrm{SC} / \mathrm{BR}$ & -27.2000 & -53.4170 & $770 \pm 100$ & 1278 & C14 & SI-439 & $\begin{array}{l}\text { Piazza 1969, Mielke and Long } \\
1969\end{array}$ \\
\hline SC-VP-38 & SC-VP-38 & $\mathrm{SC} / \mathrm{BR}$ & -27.2710 & -52.5080 & $590 \pm 100$ & 1392 & C14 & SI-826 & $\begin{array}{l}\text { Miller } 1971, \text { Stuckenrath and } \\
\text { Mielke } 1973\end{array}$ \\
\hline Joaquim Bosquero & SC-U-71 & $\mathrm{SC} / \mathrm{BR}$ & -27.4984 & -51.7500 & $900 \pm 50$ & 1184 & $\mathrm{C} 14$ & Beta-118377 & $\begin{array}{l}\text { Projeto Machadinho } 1988 \text { in } \\
\text { Noelli } 1999-2000\end{array}$ \\
\hline José Vieira & SC-U-368 & $\mathrm{SC} / \mathrm{BR}$ & -27.5330 & -51.7676 & $420 \pm 60$ & 1526 & C14 & Beta-118376 & $\begin{array}{l}\text { Projeto Machadinho } 1988 \text { in } \\
\text { Noelli 1999-2000 }\end{array}$ \\
\hline José Vieira & SC-U-368 & $\mathrm{SC} / \mathrm{BR}$ & -27.5330 & -51.7676 & $530 \pm 70$ & 1431 & C14 & Beta-118375 & $\begin{array}{l}\text { Projeto Machadinho } 1988 \text { in } \\
\text { Noelli 1999-2000 }\end{array}$ \\
\hline Tapera & SC-LF-2/FLN-58 & $\mathrm{SC} / \mathrm{BR}$ & -27.5940 & -48.5010 & $550 \pm 70$ & 1418 & C14 & SI-244 & $\begin{array}{l}\text { Rohr 1969, Long and Mielke } \\
1967\end{array}$ \\
\hline Morro Bonito 2 & - & $\mathrm{SC} / \mathrm{BR}$ & -28.6089 & -48.9839 & $430 \pm 40$ & 1495 & $\mathrm{C} 14$ & Beta-262754 & Milheira and De Blasis, 2011 \\
\hline Morro Bonito 3 & - & $\mathrm{SC} / \mathrm{BR}$ & -28.6130 & -48.9918 & $510 \pm 40$ & 1437 & $\mathrm{C} 14$ & Beta-262755 & Milheira and De Blasis, 2011 \\
\hline Morro Bonito 1 & - & $\mathrm{SC} / \mathrm{BR}$ & -28.6170 & -49.8842 & $520 \pm 50$ & 1433 & $\mathrm{C} 14$ & Beta-262753 & Milheira and De Blasis, 2011 \\
\hline Laranjal 1 & - & $\mathrm{SC} / \mathrm{BR}$ & -28.6201 & -48.9380 & $440 \pm 40$ & 1486 & $\mathrm{C} 14$ & Beta-262751 & Milheira and De Blasis, 2011 \\
\hline Sibelco & - & $\mathrm{SC} / \mathrm{BR}$ & -28.6213 & -48.9991 & $550 \pm 60$ & 1419 & $\mathrm{C} 14$ & Beta-262752 & Milheira and De Blasis 2011 \\
\hline Arroio Corrente 5 & - & $\mathrm{SC} / \mathrm{BR}$ & -28.6852 & -49.0359 & $470 \pm 40$ & 1459 & $\mathrm{C} 14$ & Beta-280654 & Milheira and De Blasis, 2011 \\
\hline OlhoD'água 1 & - & $\mathrm{SC} / \mathrm{BR}$ & -28.7917 & -49.1972 & $560 \pm 40$ & 1417 & $\mathrm{C} 14$ & Beta-280652 & Milheira and De Blasis, 2011 \\
\hline $\begin{array}{l}\text { Aldeia do Cemiterio } \\
\text { Lagoa dos Esteves }\end{array}$ & - & $\mathrm{SC} / \mathrm{BR}$ & -28.8412 & -49.2956 & $720 \pm 70$ & 1260 & $\mathrm{TL}$ & FATEC & Lino, 2007 \\
\hline $\begin{array}{l}\text { Aldeia da Lagoa Mae } \\
\text { Luzia }\end{array}$ & - & $\mathrm{SC} / \mathrm{BR}$ & -28.8659 & -49.3231 & $610 \pm 70$ & 1370 & $\mathrm{TL}$ & FATEC & Lino, 2007 \\
\hline Poço Grande & - & $\mathrm{SC} / \mathrm{BR}$ & -26.4446 & -48.8438 & $340 \pm 35$ & 1640 & $\mathrm{TL}$ & - & Farias and Kneips, 2010 \\
\hline Aldeia da ZPE & - & $\mathrm{SC} / \mathrm{BR}$ & -28.2166 & -48.6967 & $810 \pm 85$ & 1170 & $\mathrm{TL}$ & - & Farias and Kneips, 2010 \\
\hline Aldeia da ZPE & - & $\mathrm{SC} / \mathrm{BR}$ & -28.2166 & -48.6967 & $715 \pm 75$ & 1265 & $\mathrm{TL}$ & - & Farias and Kneips, 2010 \\
\hline Jaboticaba 1 & RS-VZ-41 & $\mathrm{RS} / \mathrm{BR}$ & -27.1670 & -53.7330 & $225 \pm 50$ & 1758 & C14 & SI-701 & $\begin{array}{l}\text { Miller 1969, Stuckenrath and } \\
\text { Mielke } 1973\end{array}$ \\
\hline Três Bocas 2 & - & $\mathrm{RS} / \mathrm{BR}$ & -27.5290 & -54.6560 & $410 \pm 60$ & 1536 & $\mathrm{C} 14$ & LP-1761 & Costa Angrizani, 2012 \\
\hline Itajubá 1 & - & $\mathrm{RS} / \mathrm{BR}$ & -27.5440 & -54.6680 & $390 \pm 60$ & 1548 & $\mathrm{C} 14$ & LP-1751 & Costa Angrizani, 2012 \\
\hline Barra do Sto. Cristo 1 & - & $\mathrm{RS} / \mathrm{BR}$ & -27.5680 & -54.7200 & $500 \pm 70$ & 1452 & $\mathrm{C} 14$ & LP-1874 & Costa Angrizani, 2012 \\
\hline Ilha Comandaí & RS-VZ-12 & $\mathrm{RS} / \mathrm{BR}$ & -27.8000 & -55.1000 & $215 \pm 105$ & 1753 & $\mathrm{C} 14$ & SI-702 & Stuckenrath and Mielke 1973 \\
\hline Adolfo Schenkel & RS-C-63 & $\mathrm{RS} / \mathrm{BR}$ & -29.3720 & -51.5650 & $190 \pm 85$ & Modern*** & $\mathrm{C} 14$ & SI-1197 & Ribeiro 1968, 1974, Rogge 2005 \\
\hline Lincoln Steuernagel & RS-MJ-53a & $\mathrm{RS} / \mathrm{BR}$ & -29.4170 & -53.3320 & $905 \pm 95$ & 1165 & $\mathrm{C} 14$ & SI-1196 & Brochado 1969, 1973 \\
\hline Eri Rampellotto & RS-MJ-71 & $\mathrm{RS} / \mathrm{BR}$ & -29.5320 & -53.5360 & $265 \pm 90$ & 1694 & $\mathrm{C} 14$ & SI-2199 & Brochado 1971, 1984 \\
\hline Pedra Grande & RS-SM-7 & $\mathrm{RS} / \mathrm{BR}$ & -29.5500 & -54.2500 & $605 \pm 40$ & 1392 & $\mathrm{C} 14$ & SI-1002 & $\begin{array}{l}\text { Brochado } 1969, \text { Stuckenrath } \\
\text { and Mielke } 1973\end{array}$ \\
\hline Pedra Grande & RS-SM-7 & $\mathrm{RS} / \mathrm{BR}$ & -29.5500 & -54.2500 & $800 \pm 40$ & 1257 & C14 & SI-1003 & $\begin{array}{l}\text { Brochado } 1969, \text { Stuckenrath } \\
\text { and Mielke } 1973\end{array}$ \\
\hline Bom Jardim Velho & RS-C-14 & $\mathrm{RS} / \mathrm{BR}$ & -29.6289 & -51.3729 & $745 \pm 115$ & 1294 & $\mathrm{C} 14$ & SI-1198 & Ribeiro 1968, 1974 \\
\hline Arnildo Drew & RS-MJ-87 & $\mathrm{RS} / \mathrm{BR}$ & -29.6780 & -53.6440 & $695 \pm 55$ & 1340 & $\mathrm{C} 14$ & SI-2200 & Brochado 1971, 1984 \\
\hline Elias Dal Prá & RS-MJ-50 & $\mathrm{RS} / \mathrm{BR}$ & -29.6890 & -53.5500 & $110 \pm 100$ & Modern*** & $\mathrm{C} 14$ & SI-817 & $\begin{array}{l}\text { Brochado } 1969, \text { Stuckenrath } \\
\text { and Mielke } 1973\end{array}$ \\
\hline Elias Dal Prá & RS-MJ-50 & $\mathrm{RS} / \mathrm{BR}$ & -29.6890 & -53.5500 & $354 \pm 105$ & 1586 & C14 & SI-818 & $\begin{array}{l}\text { Brochado 1971, Stuckenrath } \\
\text { and Mielke } 1973\end{array}$ \\
\hline Reinaldo Kappel & RS-RP-140 & $\mathrm{RS} / \mathrm{BR}$ & -29.7290 & -52.6810 & $180 \pm 60$ & Modern*** & $\mathrm{C} 14$ & SI-3523 & Ribeiro 1991 \\
\hline Aurélio C. Bernardes & RS-MJ-90 & $\mathrm{RS} / \mathrm{BR}$ & -29.7290 & -52.6810 & $220 \pm 85$ & 1758 & $\mathrm{C} 14$ & SI-2202 & Brochado 1971, 1984 \\
\hline Jerônimo Rodrigues & RS-MJ-98 & $\mathrm{RS} / \mathrm{BR}$ & -29.7610 & -53.2490 & $775 \pm 65$ & 1278 & $\mathrm{C} 14$ & SI-2198 & Brochado 1971, 1984 \\
\hline
\end{tabular}


Table 4 (continued)

\begin{tabular}{|c|c|c|c|c|c|c|c|c|c|}
\hline Site & $\begin{array}{l}\text { Identification } \\
\text { code }\end{array}$ & $\begin{array}{l}\text { State/ } \\
\text { country }\end{array}$ & Latitude & Longitude & Age years BP & $\begin{array}{l}\text { Age years } \\
\mathrm{AD}\end{array}$ & Method & Lab code & Reference \\
\hline Bassani & RS-M-35 & $\mathrm{RS} / \mathrm{BR}$ & -29.7670 & -50.0830 & $870 \pm 100$ & 1194 & $\mathrm{C} 14$ & SI-412 & $\begin{array}{l}\text { Miller 1967, Stuckenrath and } \\
\text { Mielke } 1970\end{array}$ \\
\hline Bassani & RS-M-35 & $\mathrm{RS} / \mathrm{BR}$ & -29.7670 & -50.0830 & $1070 \pm 110$ & 1018 & $\mathrm{C} 14$ & SI-413 & $\begin{array}{l}\text { Miller 1967, Stuckenrath and } \\
\text { Mielke } 1970\end{array}$ \\
\hline João B. Cantarelli & RS-MJ-42 & $\mathrm{RS} / \mathrm{BR}$ & -29.8240 & -53.3830 & $130 \pm 105$ & Modern*** & $\mathrm{C} 14$ & SI-815 & $\begin{array}{l}\text { Brochado 1969, Stuckenrath } \\
\text { and Mielke } 1973\end{array}$ \\
\hline Arnaldo M. Silva & RS-MJ-47 & $\mathrm{RS} / \mathrm{BR}$ & -29.8500 & -53.3800 & $530 \pm 120$ & 1442 & $\mathrm{C} 14$ & SI-816 & $\begin{array}{l}\text { Brochado } 1971 \text {, Stuckenrath } \\
\text { and Mielke } 1973\end{array}$ \\
\hline Paso Fundo & RS-M-16 & $\mathrm{RS} / \mathrm{BR}$ & -29.9330 & -50.2170 & $520 \pm 200$ & 1476 & $\mathrm{C} 14$ & SI-410 & $\begin{array}{l}\text { Miller 1967, Stuckenrath and } \\
\text { Mielke } 1970\end{array}$ \\
\hline Paso Fundo & RS-M-16 & $\mathrm{RS} / \mathrm{BR}$ & -29.9330 & -50.2170 & $540 \pm 100$ & 1429 & $\mathrm{C} 14$ & SI-411 & $\begin{array}{l}\text { Miller 1967, Stuckenrath and } \\
\text { Mielke } 1970\end{array}$ \\
\hline Santa Rita & RS-SR-342 & $\mathrm{RS} / \mathrm{BR}$ & -29.9810 & -51.5330 & $420 \pm 60$ & 1526 & $\mathrm{C} 14$ & Beta-118376 & Hilbert 1999 \\
\hline Santa Rita & RS-SR-342 & $\mathrm{RS} / \mathrm{BR}$ & -29.9810 & -51.5330 & $530 \pm 70$ & 1431 & C14 & Beta-118375 & Hilbert 1999 \\
\hline Ilha Francisco Manuel & RS-C-71 & $\mathrm{RS} / \mathrm{BR}$ & -30.2653 & -51.1632 & $610 \pm 50$ & 1349 & $\mathrm{C} 14$ & Beta & Gaulier 2001-2002 \\
\hline Lagoa da Porteira 1 & RS-LC-80 & $\mathrm{RS} / \mathrm{BR}$ & -30.4310 & -50.6050 & $280 \pm 50$ & 1654 & $\mathrm{C} 14$ & Beta-202366 & Schmitz and Sandrin 2009 \\
\hline Lagoa da Porteira 1 & RS-LC-80 & $\mathrm{RS} / \mathrm{BR}$ & -30.4310 & -50.6050 & $563 \pm 45$ & 1417 & $\mathrm{TL}$ & LVD-655 & Schmitz and Sandrin 2009 \\
\hline Bento Prestes & RS-CM-11 & $\mathrm{RS} / \mathrm{BR}$ & -30.8450 & -52.8920 & $445 \pm 40$ & 1482 & $\mathrm{C} 14$ & SI-6402 & Ribeiro et al., 1986 \\
\hline Ribes & PS-G-PA-4 & $\mathrm{RS} / \mathrm{BR}$ & -31.5030 & -52.4050 & $510 \pm 70$ & 1470 & $\mathrm{TL}$ & FATEC-1968 & Milheira 2008 \\
\hline Camping & PS-02 & $\mathrm{RS} / \mathrm{BR}$ & -31.7120 & -52.1740 & $380 \pm 50$ & 1552 & $\mathrm{C} 14$ & Beta-234205 & Milheira 2008 \\
\hline Totó & PS-03 & $\mathrm{RS} / \mathrm{BR}$ & -29.7610 & -53.2490 & $530 \pm 40$ & 1429 & $\mathrm{C} 14$ & Beta-237665 & Milheira 2008 \\
\hline Fazenda Soares & RS-RG-002 & $\mathrm{RS} / \mathrm{BR}$ & -31.8470 & -52.2220 & $510 \pm 60$ & 1441 & C14 & Beta-64284 & $\begin{array}{l}\text { Hilbert et al., } 1997 \text { in Carle } \\
2002\end{array}$ \\
\hline Fazenda Soares & RS-RG-002 & $\mathrm{RS} / \mathrm{BR}$ & -31.8470 & -52.2220 & $580 \pm 50$ & 1405 & C14 & Beta-64560 & $\begin{array}{l}\text { Hilbert et al., } 1997 \text { en Carle } \\
2002\end{array}$ \\
\hline Fazenda Soares & RS-RG-002 & $\mathrm{RS} / \mathrm{BR}$ & -31.8470 & -52.2220 & $890 \pm 40$ & 1197 & C14 & SI-1190 & $\begin{array}{l}\text { Naue } 1973, \text { Brochado } 1984, \\
\text { Carle } 2002\end{array}$ \\
\hline RS-T-114 & RS-T-114 & $\mathrm{RS} / \mathrm{BR}$ & -29.2885 & -52.1195 & $592 \pm 67$ & 1388 & $\mathrm{TL}$ & LACIFID-USP & Kreutz 2008 \\
\hline
\end{tabular}

Table 5

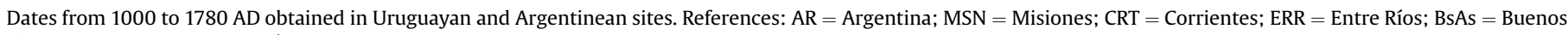
Aires; UR = Uruguay; RN = Río Negro.

\begin{tabular}{|c|c|c|c|c|c|c|c|c|}
\hline Site & $\begin{array}{l}\text { Province or } \\
\text { department/ } \\
\text { Country }\end{array}$ & Latitude & Longitude & Age years BP & Age years AD & Method & Lab code & Reference \\
\hline Panambí 3 & MSN/AR & -27.6920 & -54.9010 & $920 \pm 70$ & 1158 & C14 & LP-176 & Poujade 1994,Sempé and Caggiano 1995 \\
\hline 519 & CRT/AR & -27.3560 & -56.1690 & $410 \pm 50$ & 1534 & C14 & B-197133 & Rodríguez 2009 \\
\hline 516 & CRT/AR & -27.4150 & -56.2690 & $380 \pm 50$ & 1552 & C14 & B-197132 & Rodríguez 2009 \\
\hline González & CRT/AR & -27.5060 & -56.7290 & $420 \pm 50$ & 1519 & $\mathrm{C} 14$ & B-105251 & Rodríguez 2009 \\
\hline San Antonio & CRT/AR & -27.5140 & -56.7380 & $610 \pm 70$ & 1374 & C14 & B-105247 & Rodríguez 2009 \\
\hline Valenzuela & CRT/AR & -27.5200 & -56.7300 & $300 \pm 50$ & 1623 & C14 & B-105250 & Rodríguez 2009 \\
\hline Valenzuela & CRT/AR & -27.5200 & -56.7300 & $810 \pm 60$ & 1250 & C14 & B-197128 & Rodríguez 2009 \\
\hline Medina I & CRT/AR & -27.5520 & -57.1490 & $300 \pm 50$ & 1623 & C14 & Beta-105248 & Rodríguez 2009 \\
\hline Medina I & CRT/AR & -27.5520 & -57.1490 & $330 \pm 50$ & 1571 & C14 & LP-750 & Rodríguez 1997, 2009 \\
\hline Medina I & CRT/AR & -27.5520 & -57.1490 & $360 \pm 60$ & 1560 & C14 & Beta-105253 & Rodríguez 2009 \\
\hline Medina I & CRT/AR & -27.5520 & -57.1490 & $430 \pm 50$ & 1504 & $\mathrm{C} 14$ & LP-734 & Rodríguez 1997, 2009 \\
\hline Santa Tecla I & CRT/AR & -27.6370 & -56.6150 & $240 \pm 50$ & 1749 & $\mathrm{C} 14$ & Beta-105252 & Rodríguez 2009 \\
\hline Santa Tecla I & CRT/AR & -27.6370 & -56.6150 & $310 \pm 50$ & 1591 & C14 & Beta-197131 & Rodríguez 2009 \\
\hline Santa Tecla I & CRT/AR & -27.6370 & -56.6150 & $390 \pm 60$ & 1548 & C14 & Beta-197130 & Rodríguez 2009 \\
\hline Santa Tecla I & CRT/AR & -27.6370 & -56.6150 & $570 \pm 50$ & 1410 & C14 & B-197129 & Rodríguez 2009 \\
\hline Santa Tecla I & CRT/AR & -27.6370 & -56.6150 & $684 \pm 170$ & 1329 & C14 & Ingeis-1338 & Rodríguez 1997, 2009 \\
\hline Llamarada I & CRT/AR & -28.3190 & -58.0820 & $370 \pm 60$ & 1556 & C14 & Beta-41943 & Mujica 1995b, 1995c \\
\hline Llamarada I & CRT/AR & -28.3190 & -58.0820 & $400 \pm 50$ & 1543 & $\mathrm{C} 14$ & Beta-41939 & Mujica 1995b, 1995c \\
\hline Llamarada I & CRT/AR & -28.3190 & -58.0820 & $480 \pm 50$ & 1457 & C14 & Beta-41944 & Mujica 1995b, 1995c \\
\hline Llamarada I & CRT/AR & -28.3190 & -58.0820 & $500 \pm 60$ & 1447 & $\mathrm{C} 14$ & Beta-41940 & Mujica 1995b, 1995c \\
\hline Llamarada I & CRT/AR & -28.3190 & -58.0820 & $510 \pm 50$ & 1438 & $\mathrm{C} 14$ & Beta-41945 & Mujica 1995b, 1995c \\
\hline Llamarada I & CRT/AR & -28.3190 & -58.0820 & $520 \pm 50$ & 1433 & $\mathrm{C} 14$ & Beta-41942 & Mujica 1995b, 1995c \\
\hline Llamarada I & CRT/AR & -28.3190 & -58.0820 & $580 \pm 50$ & 1405 & C14 & Beta-41941 & Mujica 1995b, 1995c \\
\hline Ensenada del Bellaco & ERR/AR & -33.0915 & -58.4349 & $526 \pm 45$ & 1407 & C14 & AA-103895 & This paper \\
\hline Cem.A $A^{\circ}$ Paicarabí y Fredes & BsAs/AR & -34.2289 & -58.5924 & $421 \pm 45$ & 1474 & C14 & AA-103896 & This paper \\
\hline Arroyo Fredes & BsAs/AR & -34.2090 & -58.5680 & $370 \pm 50$ & 1556 & $\mathrm{C} 14$ & LP-1428 & Loponte et al., 2011 \\
\hline Arroyo Fredes & BsAs/AR & -34.2090 & -58.5680 & $402 \pm 40$ & 1540 & C14 & AA-77309 & Loponte et al., 2011 \\
\hline Arroyo Fredes & BsAs/AR & -34.2090 & -58.5680 & $690 \pm 70$ & 1339 & $\mathrm{C} 14$ & UGA-10789 & Loponte et al., 2011 \\
\hline Arroyo La Glorieta & BsAs/AR & -34.3460 & -58.7440 & $416 \pm 41$ & 1514 & $\mathrm{C} 14$ & AA-93216 & Bonomo et al., 2011 \\
\hline Arroyo Malo & BsAs/AR & -34.3060 & -58.6984 & $442 \pm 45$ & 1489 & $\mathrm{C} 14$ & AA-103897 & This paper \\
\hline El Arbolito & BsAs/AR & -34.1270 & -58.2600 & $405 \pm 35$ & 1531 & $\mathrm{C} 14$ & GrN-5146 & Cigliano 1968 \\
\hline Arroyo Negro & RN/URU & -32.4970 & -58.1890 & $590 \pm 50$ & 1390 & $\mathrm{TL}$ & UCTL-1673 & Farías Gluchy 2005 \\
\hline Isla Viscaíno (Pirata) & RN/URU & -33.4260 & -58.4250 & $790 \pm 105$ & 1260 & C14 & URU-0118 & Castillo 2004 \\
\hline Isla Viscaíno (Pirata) & RN/URU & -33.4260 & -58.4250 & $870 \pm 100$ & 1194 & $\mathrm{C} 14$ & URU-0118 & Castillo 2004 \\
\hline Isla Viscaíno (Pirata) & RN/URU & -33.4260 & -58.4250 & $1020 \pm 130$ & 1089 & C14 & URU-0117 & Castillo 2004 \\
\hline
\end{tabular}




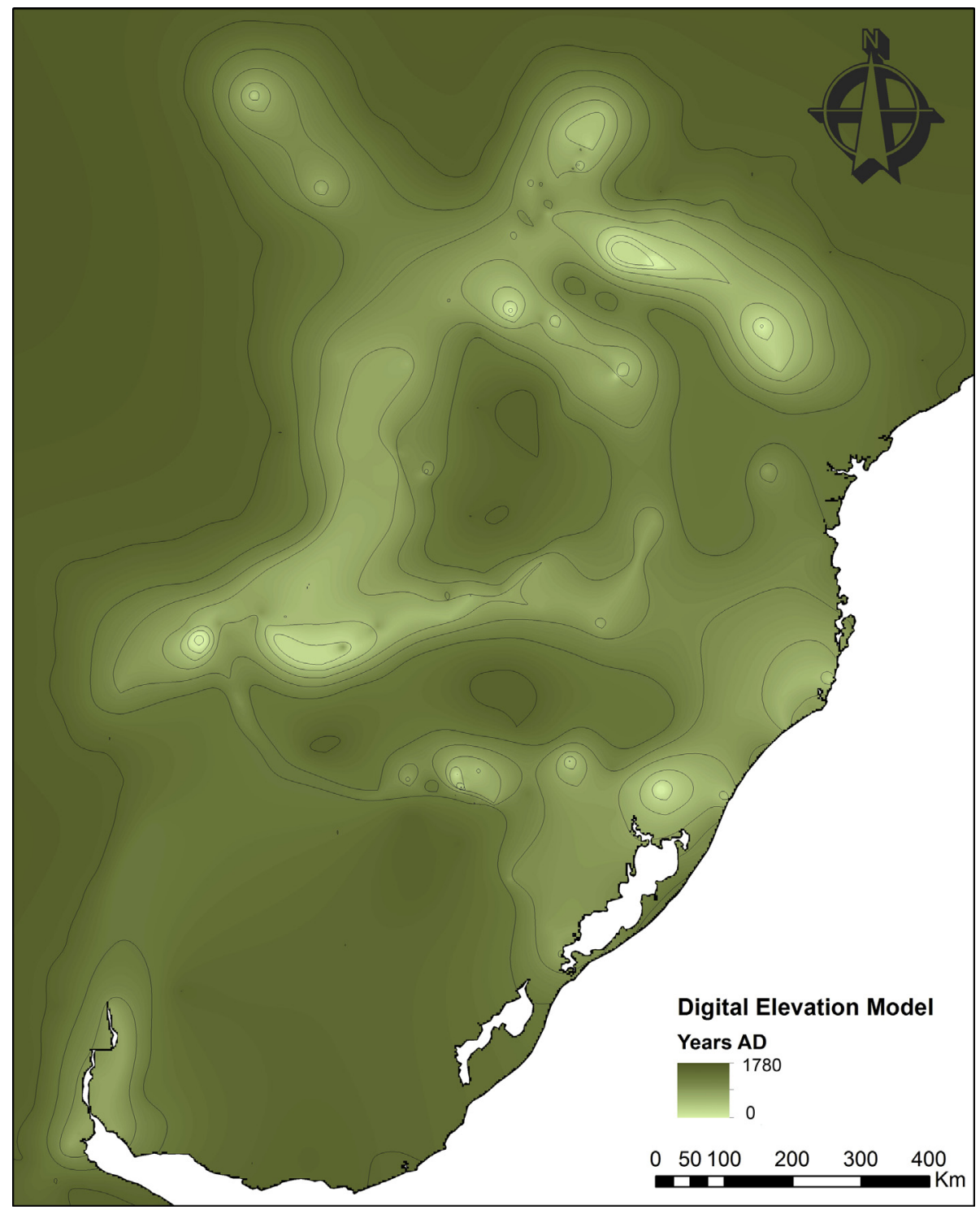

Fig. 3. Digital Elevation Model (DEM).

\subsection{Temporal Visualization Model (TVM)}

The TVM, which considers the temporal variables in addition to the spatial variables, provided the possibility of creating a chronological sequence for these dispersion routes. From the digital animation of the TVM, it was possible to display the expansion timing on one year intervals. This allowed defining the expansion rates, divided into discrete periods of major expansion pulses (principally new sites in new areas) or periods of relative stability in the previous occupied areas (principally new sites in the same area). It was also possible to establish when the Guaraní are present in distant zones of the La Plata Basin.

On the basis of the collected data, between the year 0 and 300 $\mathrm{AD}$ the earliest evidence of unambiguous Guaraní settlements appears in the La Plata Basin (Table 2). The earliest date comes from the confluence area of the Paraná and the Iguaçu (or Iguazú) Rivers, Fazenda Dona Carlota 2 archaeological site, dated 27 AD. Around 200 AD, the Guaraní presence is recorded along the Paraná River, about $100 \mathrm{~km}$ south from its confluence with the Verde River (Lagoa 1 archaeological site), in the Itararé River of the Paranapanema basin (Acampamento Três site) and in northern
Corrientes (San Miguel II site). Towards the end of this first interval, two new sites are recorded: the first in the watershed between the Ibucuí and Jacuí Rivers in Rio Grande do Sul state (Emilio Kieffer site), and the second one in the Ivaí River (João Batista site). The six archaeological sites occupied during the first three centuries of our Era suggest the first significant pulse of the Guaraní territorial expansion (Fig. 5). However, given the fact that there are only six archeological sites with a single date, spatial and chronological changes in this first pulse are expected, as new dates are obtained.

During the following seven centuries, between 300 and 1000 $\mathrm{AD}$, on the basis of 24 archaeological sites with 34 numerical dates, it is possible to observe a period of relative stability, which is a moment of permanence in the already occupied areas and only a very limited dispersal towards new areas (Table 3 and Fig. 6). It was possible to record an increase in the number of settlements in the same areas that present the earliest dates: the Paranapanema Basin (five sites with five dates between 338 and 947 AD), the confluence of the Paraná and Iguaçú Rivers (four sites with dates between 479 and $686 \mathrm{AD}$ ), the Paraná River between the mouths of the Ivinhema and the Verde Rivers (six sites with 13 dates between 380 and 1000 AD), the Ivaí Basin (three sites with three new dates between 461 


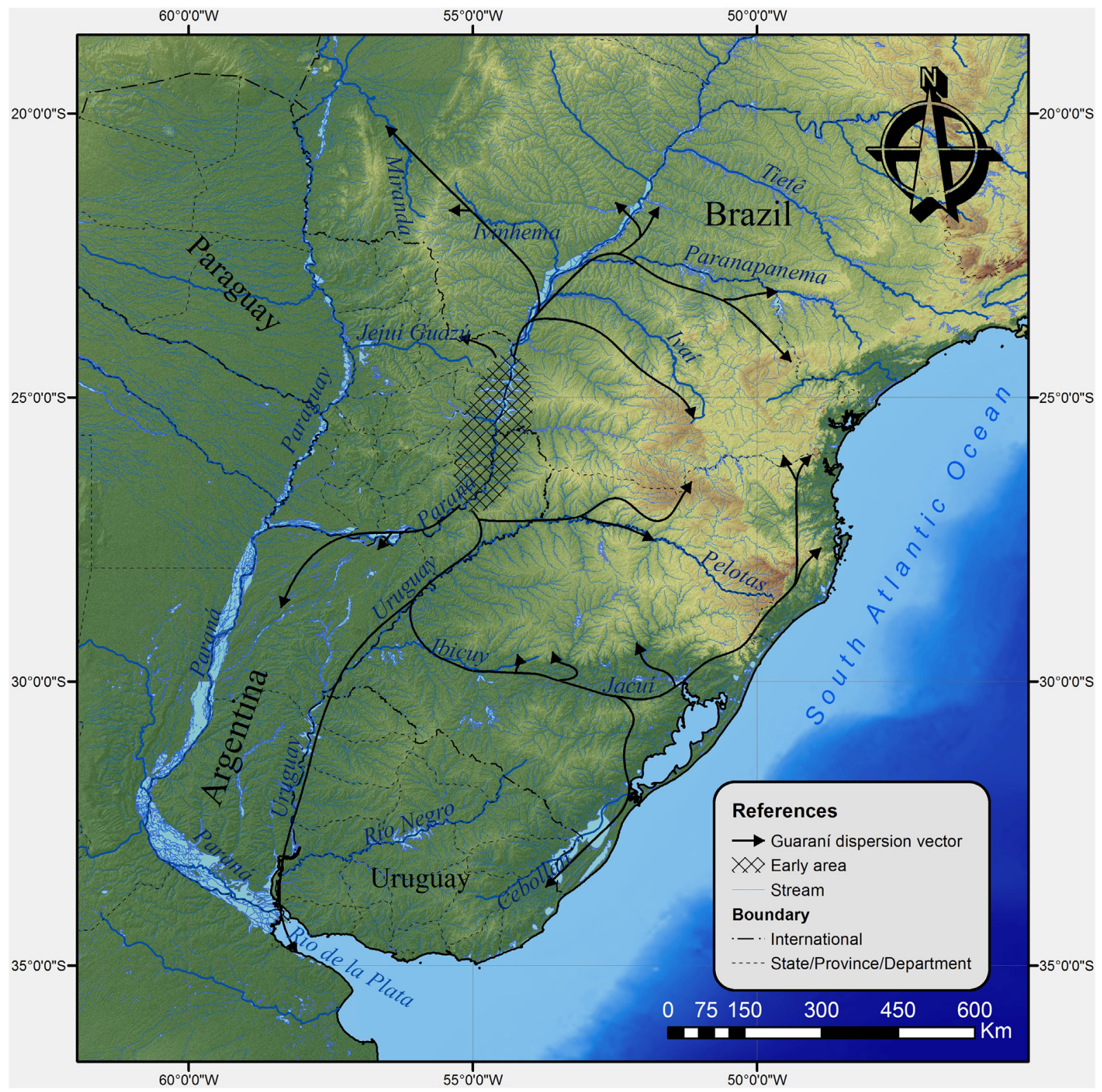

Fig. 4. Dispersion Routes Model (DRM).

and 717 AD), the Uruguay River (one site with an 859 AD date) and the middle basin of the Jacuí (two sites with three dates between 610 and $906 \mathrm{AD}$ ).

The new areas occupied are restricted to the most southern sectors, and it is possible to observe an advance towards the east, represented by the dates from the RS-T- 114 and Palmeira 2 sites, in the Sinos River basin (near the Atlantic coast of Rio Grande do Sul), between 570 and 718 AD. Finally, towards the end of the first millennium of our Era, the occupation of the Atlantic coast seems to have started. This is supported by the three dates between 930 and $980 \mathrm{AD}$ from the Aldeia ZPE archaeological site, although additional data are needed to define the moment of arrival at the littoral zone, which represents a natural limit to the Guaraní expansion.

Between 1000 and 1780 AD, 200 numerical dates were recorded which correspond to a total of 119 archaeological sites and 5 previously mentioned sites with European materials. As in the former period, the quantity of settlements increases in the previously occupied areas (Table 4). In this sense, there is a significant number of new dates from the mouth of the Iguaçú and the Paraná Rivers (11 new dates between 1302 and $1771 \mathrm{AD}$ corresponding to 10 sites); the course of the Paraná River between the Iguaçu and the Verde Rivers ( 75 dates corresponding to 28 sites between 1030 and 1752 AD); the Paranapanema Basin (9 dates between 1014 and 1429 AD corresponding to 8 sites); the Ivaí River ( 9 dates between 1026 and $1709 \mathrm{AD}$ corresponding to 6 six sites); the Jacuí Basin (13 dates between 1165 and $1758 \mathrm{AD}$ corresponding to 11 sites); the Upper Uruguay ( 5 dates between 1158 and 1753 corresponding to 5 sites); the Atlantic coast of Santa Catarina and Rio Grande do Sul (13 dates between 1018 and 1495 AD corresponding to 11 sites) and the north of Corrientes province ( 15 dates between 1250 and 1749 AD corresponding to 7 sites).

During this period, a notable territorial expansion into new areas also occurred (Fig. 7). It was possible to confirm the presence of archaeological sites in areas with no prior record of Guaraní occupations: the northern Atlantic coast of Santa Catarina (2 dates between 1418 and 1640 AD corresponding to the Poço Grande and 


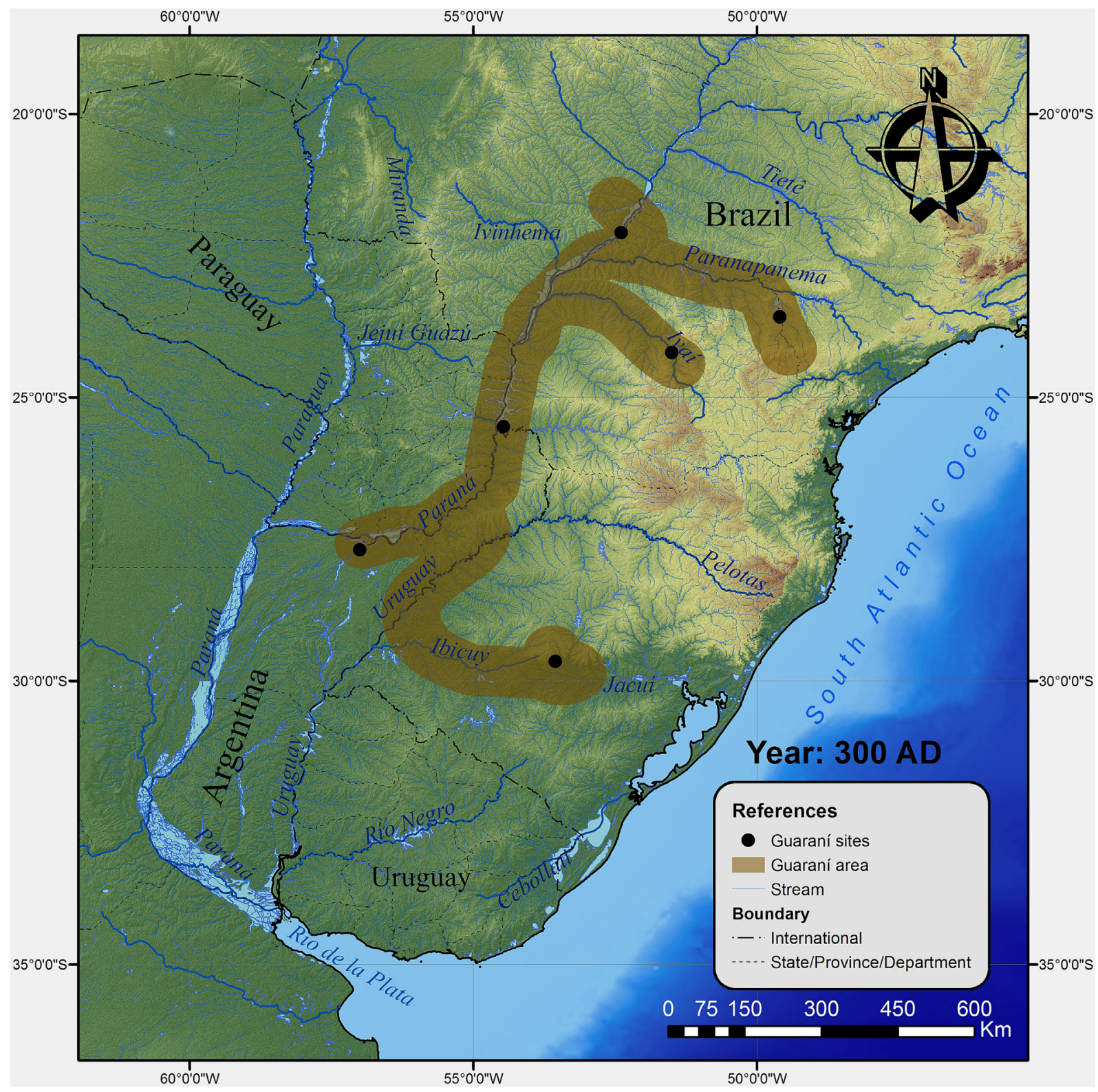

Fig. 5. Temporal Visualization Model (TVM) from 0 to $300 \mathrm{AD}$.

Tapera sites), the Miranda River and the Ivinhema River headwater (3 dates between 1111 and 1349 AD corresponding to 2 sites), the Upper Uruguay River, towards the Pelotas River (11 dates between 1019 and 1758 AD corresponding to 9 sites), the Iguaçu River, between Santa Catarina and Paraná states (3 dates of 1432 and 1455 AD corresponding to 3 sites), the Batel marshes ( 7 dates between 1405 and 1556 AD corresponding to the Llamarada I site), the Los Patos lagoon and the Camaquã basin (12 dates between 1197 and $1654 \mathrm{AD}$ corresponding to 8 sites), the Cebollatí River basin (one site with European materials), the Middle and Lower Uruguay River ( 5 dates between 1089 and 1407 AD corresponding to 3 sites, along with 2 sites with European materials), the Paraná Delta in the Lower Paraná River and the La Plata River ( 7 dates between 1339 and $1556 \mathrm{AD}$ corresponding to 5 sites, along with 2 sites with European materials).

To sum up, from 1000 AD onwards, in addition to a growing density of sites in the already occupied areas, there was a marked pulse of expansion in all directions. It is possible to observe Guaraní dispersal towards the west up to the Miranda River and towards the east, avoiding the elevated zones of the Brazilian Highlands, down to the Atlantic coast of Brazil and Uruguay. Lastly, sites along the forest galleries of the Uruguay and Paraná lower courses and in the Martín García Island and the levees of the La Plata River were recorded. The dynamics of the Guaraní mobility during this second pulse of expansion changes drastically since the beginning of the 16 th century, coinciding with the deep transformations caused by the Luso-Hispanic conquest and colonization.

\section{Discussion}

\subsection{Brochado's view}

On the basis of Lathrap's (1956) ideas, Brochado (1984) proposed that the phenomenon of the spatial dispersion of a pottery style can be attributed to three general processes: 1) the distribution of vessels from one or several production centers with the 


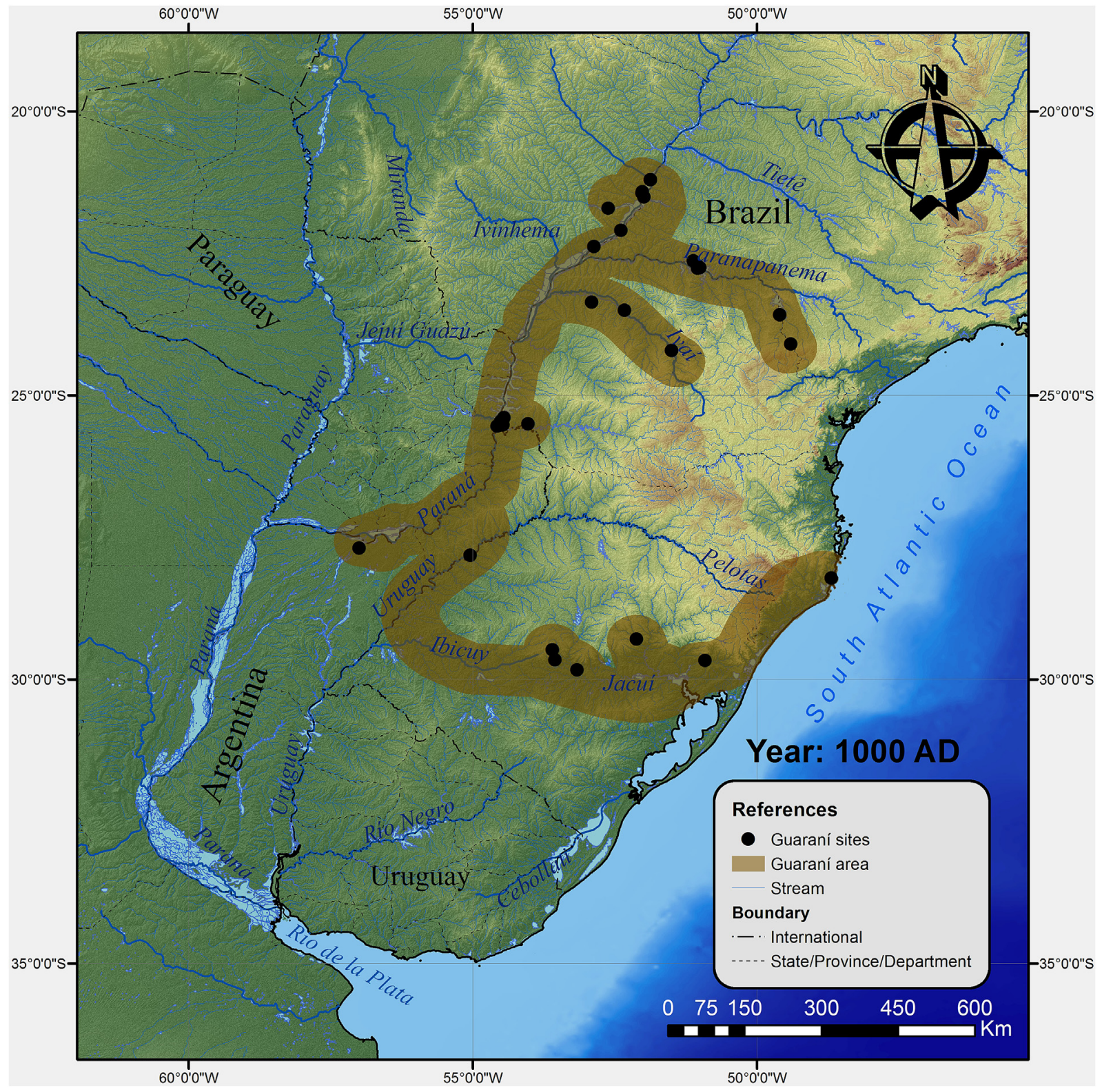

Fig. 6. Temporal Visualization Model (TVM) from 300 to 1000 AD.

purpose of supplying people who do not produce vessels; 2) the imitation or adoption by some populations who lacked pottery or who manufactured other types of vessels; 3 ) the transport through migration or expansion by an ethnic group maintaining its own pottery tradition and reproducing it in other places. In the case of Guaraní pottery, while exchanges held with other groups are not ruled out, the author observes that there is no archaeological or ethnographic evidence indicating the existence of potteryproduction centers destined to maintain a large-scale vessel distribution. Subsequently, the dispersion of pottery styles through successive copies manufactured by different ethnic groups would depend on the existence of a wide population contingent with the same needs, willing to accept the Guaraní cultural patterns and possessing the proper technology to do it. While the existence of assimilation processes is acknowledged, it also represents an implausible hypothesis to explain the full scale of the Guaraní phenomenon. In spite of the regional variations, it is possible to observe a marked uniformity in the vessels produced within the territories identified with the Guaraní historical distribution. According to Brochado (1984), the dispersion of Guaraní pottery in that sub-continental scale can only be explained through a population growth which additionally entailed the spreading of other cultural features.

In general, the model of expansion generated in this paper coincides with Brochado's proposal (1984) on Guaraní dispersion, although there are also some divergences in regards to the routes followed and their chronologies. The results obtained here strongly support Brochado (1989) and Noelli's (1998) idea that this phenomenon consisted of a population expansion rather than a migration. Following Lathrap (1956), both authors propose that the continuous demographic growth supported by agriculture caused the villages' fission and the subsequent Guaraní search of new lands to cultivate at the periphery of their domains. The Guaraní settlement system comprised networks of villages interconnected by political, economic and social alliances. The division of the villages at the same pace of demographic growth enabled dominance over 


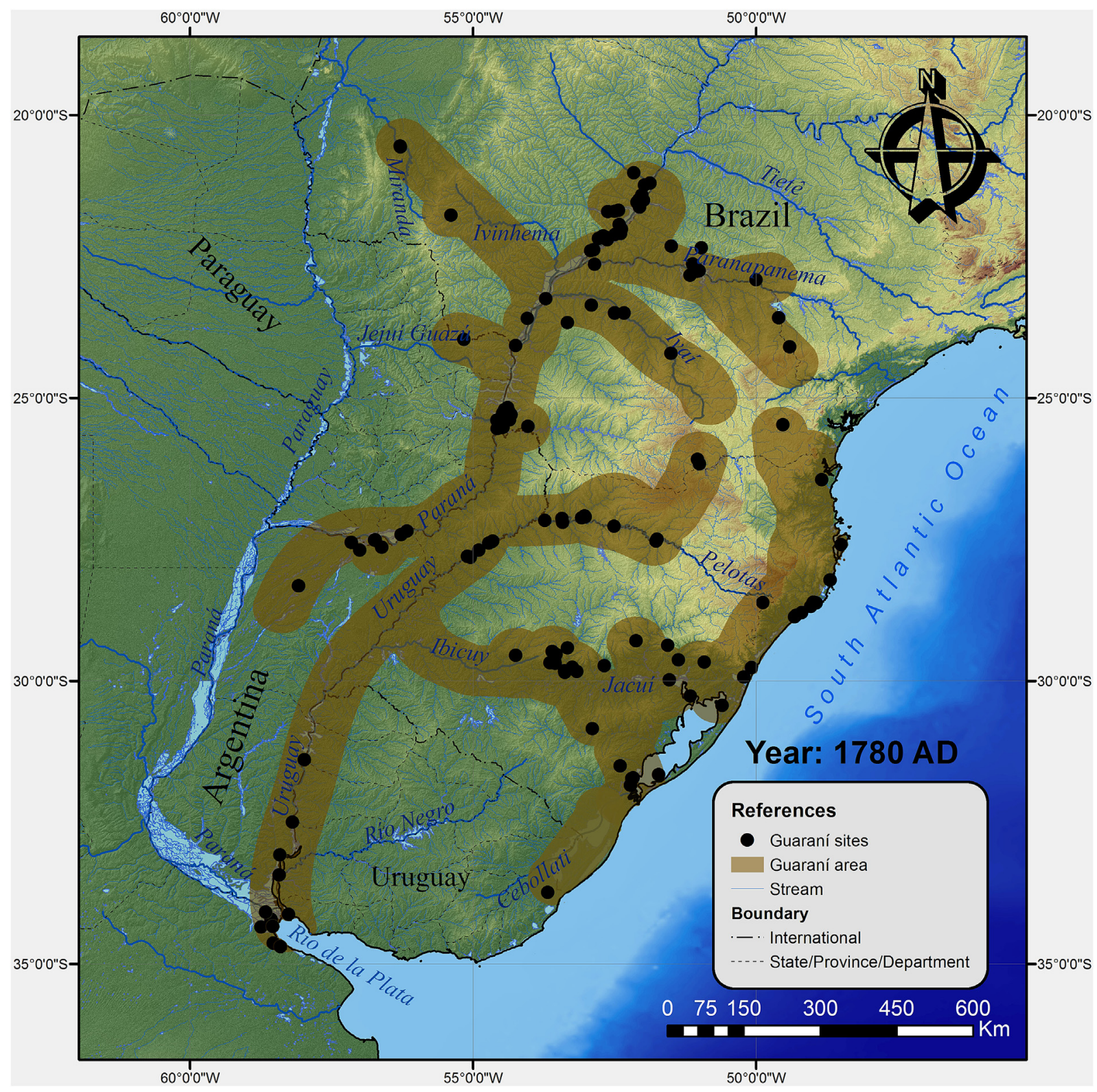

Fig. 7. Temporal Visualization Model (TVM) form 1000 to $1780 \mathrm{AD}$.

new lands without leaving the old ones. Isolated villages were nonexistent, because at the same time that the Guaraní conquered new lands they kept their previously populated territories as well. This system of village networks suggests that wherever there are ancient dates, it should be possible to obtain later dates as well.

The results presented here show that once the first Guaraní settlements were established in different areas of the La Plata Basin. There was continuity in the occupation of these places, in some cases even the same sites over hundreds of years. This is clearly evidenced by the successive occupations in the Upper Paraná River (e.g. near the current Itaipú dam) and in the Paranapanema, Ibicuí and Jacuí Rivers, where the regional network systems lasted until the demographic collapse following the 16th and 17th centuries. Therefore, the movement of the Guarani settlements responded more to an expansion of their frontiers, rather than to migratory waves of whole populations abandoning the older areas -a dynamic which better characterizes the historic migrations to the land without Evil (ywy mara ë̈ in Guaraní) (Métraux, 1927). It is also clear that this phenomenon of people movement presented great temporal depth, developing throughout almost two millennia or more. This antiquity broadly coincides with what Brochado (1984) claimed on the basis of 54 dates and the glotto-chronological dating by Rodrigues (1964).

Brochado (1984) sought to reproduce the Guaraní expansion in Eastern South America through the connections between the earliest available dates. In order to achieve that and following the premise that the larger rivers served as water routes and rich sources of staple resources, he mapped the Guaraní movements (Fig. 8a). Brochado claims that the early dispersion occurred between 100 and $200 \mathrm{AD}$, when the Guarani already occupied (although sparsely) the area comprised between the Ivaí and the Jacuí Rivers. On the basis of the dates arranged in intervals of 200 years, he proposes that these populations dispersed through various fluvial and coastal routes: down the Paraguay River up to the Middle Paraná River (between 200 and 400 AD); through the Upper Paraná, Upper Uruguay and the center of the Rio Grande do 


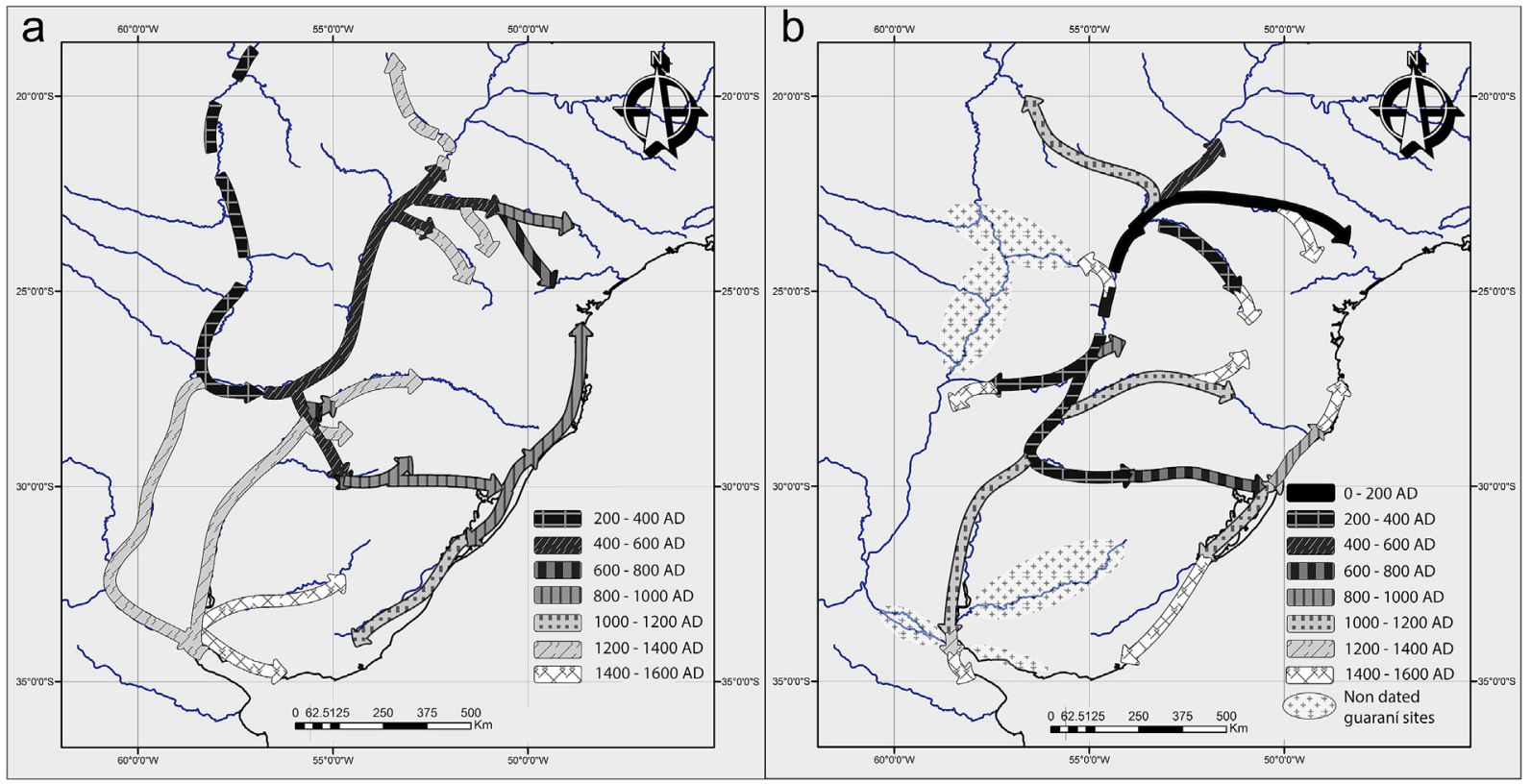

Fig. 8. Comparison between Brochado's model (a; modified from Brochado's 1984: Figure 15) and the expansion model proposed here (b).

Sul state (between 400 and 600 AD); upstream Uruguay and Paranapanema Rivers (600-800 AD); from the Jacuí basin towards the Atlantic coast and, from there, north to São Paulo state and south to the Los Patos lagoon (800-1000 AD); skirting the Atlantic coast heading south (1000-1200 AD); upstream towards Uruguay, Iguaçú and Ivaí Rivers and Paranapanema tributaries, and south along the middle and lower parts of the Uruguay and Paraná Rivers to the La Plata River (1200-1400 AD); and following the Uruguayan coast of the La Plata River and upstream along the Río Negro River (1400-1600 AD).

\subsection{The Guaraní expansion according to available archaeological dates}

The increment of numerical dates since the 1990s and the calibration of radiocarbon ages with the currently available curves, enable the analysis of the chronologies and the dispersion routes proposed by Brochado (Fig. 8a) against a broader and more precise database. As a result, it was possible to confirm that between 27 and $280 \mathrm{AD}$, there were Guaraní settlements in the area between the Paranapanema River and the Ibicuí Basin. If new dates confirmed the age of the San Miguel II site, this would also demonstrate the early Guaraní presence in the province of Corrientes, Argentina.

The available dates indicate that the Upper Paraná is the area with the earliest Guaraní occupations, with the dispersion vectors departing initially from this section of the river heading north and south (Figs. 4 and 8b). Conversely, Brochado holds the hypothesis that the entry of the Guarani populations started eastwards from the Paraguay River. While this possibility is not ruled out, due to the profuse ethnohistorical information and the numerous Guaraní vessels stored in private and public Paraguayan collections, there is currently no date of Guaraní archaeological sites in Paraguay to confirm this early dispersion route. There is no archaeological data for extended areas, such as the north of the Brazilian states of Mato Grosso do Sul, Mato Grosso, and Rondônia. These regions, along with Paraguay, hold a great potential for containing Guaraní sites, even earlier than those from the Upper Paraná. For that reason, these regions would probably be highly useful to map the first stages of the expansion, before the entry into the Paraná Basin, considering linguistic studies (Rodrigues, 1964, 1986; Urban, 1992) that point to the southwestern Amazon as a likely departure point for the Tupí languages. Corrugated, nail-incised and brushed pottery, and nail-incised urn burials have been found in Bolivian Chaco (Chuquisaca and Santa Cruz; Alconini, 2013; Pärssinen, 2003). Preliminary studies of the Bolivian evidence have started to question the idea, based on written documents, that the Guaraní reached this area only recently (Pärssinen, 2003).

The presence of Guaraní settlements between 200 and 300 AD in the middle sections of the Paranapanema and Ivaí Basins (Fig. 8b), suggests an expansion towards the east earlier than suggested by Brochado. In regard to the Atlantic coast occupation, most settlement movement seems to have occurred during the end of the first millennium AD and afterwards. This moment marks the beginning of movements in different directions throughout the occupied territory, which coincides with Brochado's suggestions for the intervals between 1000 and 1600 AD. The model presented here enables the additional postulation of new expansion routes, towards the west, through the Jejuí Guazú and Miranda Rivers, where information was unknown in the 1980s. In regards to the more southern dispersions in the La Plata Basin, the absence of dates for the Guaraní sites from the Uruguayan coast of La Plata River and from the inner Negro River (Fig. 2) hinders the determination of the vectors' directions.

From the model presented here, it is possible to infer that the arrival of the Guarani in the more southern latitudes of their geographic distribution (that is, the Lower Paraná Delta and the Argentinean coast of the La Plata River) was achieved following the course of the Uruguay River. In addition to the Uruguay River, Brochado also suggests (Fig. 8a) the Middle-Lower Paraná River as a potential access route into the La Plata River. This author suggests that the Middle Paraná River could have served as a dispersion route for the Guaraní and would represent continuity for the process started in the Paraguay River. However, the archaeological information available does not support this possibility and shows that the Middle Paraná (downstream the mouth of the Paraguay River) and the Upper Paraná Delta were densely populated by other indigenous people associated with the Goya-Malabrigo 
archaeological entity (Politis and Bonomo, 2013). The archaeological record from this section of the Paraná River (Table 1) fails to show effective Guaraní occupations. For example, in the Upper Delta, only isolated material has been recorded: a burial in a polychrome urn, four complete vessels, and a reduced number of corrugated and nail-incised sherds. With the exception of some European chronicles from the 16th century (Bonomo et al., 2011), which mention Guaraní people at the surroundings of the Sancti Spíritus Fort (1527-1529), the effective settlement of these indigenous populations are restricted to the more outer islands of the Lower Paraná Delta (Outes, 1918; Lothrop, 1932; Vignati, 1941; Loponte et al., 2011; Bonomo, 2013). Similarly, in southern Brazil and Uruguay (Brochado, 1984; Scatamacchia, 1990; Cabrera Pérez, 1994, 2011; Milheira, 2011; among others), dispersion of Guaraní materials appear in the surface and in the upper layers of sites occupied by other existing indigenous groups, as can be observed in the Middle Uruguay River, the Paraná Delta and the La Plata River (Cigliano et al., 1971; Ceruti and Crowder, 1973; Rodríguez and Rodríguez, 1985; Bonomo, 2013).

The preexistence of other indigenous people and the ecological conditions, especially the predominance of open-environment vegetation, undoubtedly redirected the Guaraní dispersion and, in some cases, hindered the effective occupation of some landscapes. The Guaraní expansion did not develop in an empty space, and the relation with local populations would have influenced this (avoidance of contact, interchange of goods, marriage and kinship, war, and captive raiding). For example, due to the predominance of the Tupinambá and other indigenous, central and northern São Paulo and its littoral were not initially occupied by the Guaraní. The occupation of these sectors started only in the 19th century by some Guaraní groups that migrated from other regions of Brazil and Paraguay. Some parts bordering their occupied territories, such as the adjacent plains of the Paraná Delta, are examples of grassland environments with fewer areas covered with dense arboreal vegetation. The Guaraní economic system was designed to function within the forest, where they systematically introduce a set of plants for food, medicinal use or as raw material, handled under the Amazon agroforestry model (Noelli, 1993; Balée, 1994). This system was considerably elastic, capable of adapting to different environments, with the presence of an arboreal canopy cover as the central conditioning ecological factor. The package included hundreds of basic components (Noelli, 1993) transported throughout their whole occupation area (corn, manioc, sweet potato, squash, beans, peanuts, medicinal plants, among others). They also incorporated species from new territories, such as the araucaria tree, yerba mate, Butia palms, and algarrobo trees, among many others. The handling of plants took place in variable environments, such as cropping areas (named rozas), paths near the houses, and on forest islands in the grasslands. Their capacity to handle and even to create artificial forest islands in the grasslands could have favored the beginning of their dispersion over the margins and adjacent plains of the La Plata River, beyond the marginal riverside forest.

\section{Conclusions}

Through examination of the location and chronology of the Guaraní archaeological sites in the La Plata Basin and the littoral of southern Brazil, it was possible to generate a hypothetical model of the dispersion routes and the temporal rates towards the Late Holocene. First, the dispersion routes showed that the flow of expansion followed the fluvial courses (the Paraná, Paranapanema, Ivaí, Piquiri, Iguaçú, Uruguay, Pelotas, Ibicuí and Jacuí rivers, along with smaller tributaries). In general, it reveals the dominant cultural patterns of the Guaraní, with movements which were oriented by travel through the clean and lineal water routes and a settlement in the rich fluvial environments, frequently surrounded by dense forest cover. Later, after consolidating the occupation of these territories, they moved on to occupy and manage the vegetation in more specific sections of the hydrographic basins, up to the interfluves.

As in many archaeological studies, the results obtained here are also affected by important sampling bias. These biases range from the trajectories of the Guaraní archeology in different countries, to a survey directionality that privileged littoral and fluvial environments, mainly large rivers disregarding smaller streams or inner zones. Most of the Guarani sites were not dated; only $12 \%$ of the sites recorded here have some sort of chronological frame. Some potential early Guaraní areas such as eastern Paraguay, Bolivia and the Brazilian states of Mato Grosso do Sul, Mato Grosso and Rondônia, need more intensive survey and systematic research. Further research may extend the chronology of the first expansion pulse proposed and change some route directions, particularly the earliest ones. Although future adjustments modify the model proposed here, it have a clear power to account the general process of the Guarani expansion, once these populations were settled in the Upper Paraná at the beginning of our Era.

The numerical dates compiled enabled the calculation of the dispersion rates, entailing a history of at least 17 centuries of continuous physical movements of people. The climatic oscillations during this period do not appear to have a strong influence on the identified pulses of Guarani expansion, which transcends dry, warm, and humid moments (Iriondo and García, 1993; Stevaux, 2000). During the expansion, thousands of kilometers were covered: to the east, the Atlantic coast in Santa Catarina $(900 \mathrm{~km}$; $930 \mathrm{AD})$; to the northwest, they reached the Miranda River (450 km; $1111 \mathrm{AD}$ ); to the southwest, they reached the Batel marshes in the province of Corrientes (400 km; $1405 \mathrm{AD}$ ); and to the south, the Paraná Delta and the La Plata River $(1200$ km; 1339 and $1531 \mathrm{AD}$ ). These limits are based on the available dates, so they will vary as research intensified, especially to the west.

Two main expansion pulses and an intermediate stability period were determined. The first pulse occurs from 0 to $300 \mathrm{AD}$, when six occupations with wide geographic distribution are detected. According to the area covered during these first three centuries, it was possible to calculate an average expansion rate of $750 \mathrm{~km}^{2}$ per year. This wide extent indicates a larger spatial division between the identified villages. Recent mitochondrial DNA studies (Marrero et al., 2007) for this area indicates that the separation between the three major Guarani partialities present in Brazil (Ñandeva, Kaiowá, M'byá) occurred prior to ca. $200 \mathrm{AD}$, coinciding with the first expansion pulse. Conversely, during the longest period, from 300 to $1000 \mathrm{AD}$, there is a period of stability and successive reoccupations in the same areas (34 numerical dates in 24 sites were recorded). Some expansion movements are observed, though they are rare. Accordingly, the rate of dispersion during this period of major stability was considerably slower: $110 \mathrm{~km}^{2}$ per year. This would indicate a shorter distance between the settlements, with higher regional demographic density which resulted in a growing branch of villages into the secondary and tertiary courses of the hydrographic network. Finally, between $1000 \mathrm{AD}$ and the European conquest, the second pulse of accelerated expansion occurs. It is possible to observe a notable increase in the number of occupations (200 dates distributed in 119 sites), in addition to a faster dispersion pace of $500 \mathrm{~km}^{2}$ per year. Possibly, the demographic growth in the previous occupied areas allowed the expansion of massive Guaraní contingents to start new colonization into farther regions dominated by non-Guaraní populations.

These regional expansion processes led to dispersed Guaraní people dominating wide portions of land in the La Plata Basin and southern Brazil. These territories were maintained by continuous communications between settlements, exchange networks, and 
political alliances, key elements to explain the notable uniformity in the material culture, language, and other aspects throughout space and time. The climax of the Guaraní population growth must have reached high numbers that researchers are not prepared to accept yet. The areas where the historical data was gathered yield high numbers at first, and then the population began to decrease in the 17th and 18th centuries, mainly due to epidemics, war, slavery, and famine. As suggested by Melià (1986), the regional projections of Guaraní people reach one million in Guairá, and three hundred thousand in the Tape (Eastern Misiones historic region). The Guaraní from the La Plata River were the first to suffer the consequences of European contact, as their number decreased drastically between 1513 and 1531 (Noelli, 2004). The issue of Guaraní demographics is still a pending topic in the South American agenda which has not been satisfactorily researched yet, posing more questions than answers.

Thirty years after the PhD thesis written by J. P. Brochado, large areas historically occupied by the Guaraní are still lacking archaeological research. However, today we have a higher number of dates from Guaraní archaeological sites which enable us to confirm their continuous occupation in some areas, estimate their dispersion rates, determine the age of their arrival at opposite ends of their territories, even in places lacking previous studies, and question some of the dispersion routes proposed in the 1980s. The model presented here should be understood as a further step which is open to improvement, aiming to broaden our knowledge on this fascinating phenomenon of large-scale Guaraní movement across a vast portion of South America.

\section{Acknowledgements}

The authors wish to thank José Brochado for the reading and making comments to the manuscript. To Luciano Prates and Cesar Méndez for their invitation to participate in this special volume and two anonymous reviewers for their useful comments, which improved earlier versions of the manuscript. To Dan Rafuse for his help with the English text. To Ângelo Corrêa for sharing the data from his doctoral thesis, which is in its final stages of production. To Eduardo Bespalez for the data on the Miranda River and to Rafael Milheira and Gustavo Peretti Wagner for the data on the littoral area of southern Brazil. To Silvia Jordan for kindly sharing her pictures on the Guarani vessels included in Fig. 1. This paper is framed within the projects "Las estructuras monticulares del Delta Superior del Paraná" (ANPCyT, PICT 0665) and "Farming, Mound Building, and Social Complexity in the Upper Delta of the Paraná River" (National Geographic Society's Committee for Research and Exploration, grant \# 9328-13). Previous versions of this paper have been presented at the VI Reunión de Teoría Arqueológica en América del Sur (2012) and the XVIII Congreso Nacional de Arqueología Argentina (2013).

\section{Appendix A. Supplementary data}

Supplementary data related to this article can be found at http:// dx.doi.org/10.1016/j.quaint.2014.10.050.

\section{References}

Alconini, S., 2013. Los Incas y los Guaraníes: conflicto, etnogénesis y fluidez social en la fortaleza de Cuzcotuyo, frontera Inca Sur-Oriental. Arqueoantropológicas 3 (3), $11-26$.

Ambrosetti, J.B., 1895. Los cementerios prehistóricos del Alto Paraná (Misiones) Boletín del Instituto Geográfico Argentino XVI, 227-263.

Anthony, D.W., 2010. The Horse, the Wheel, and Language. How Bronze-age Riders from the Eurasian Steppes Shaped the Modern World. Princeton University Press, Princeton.
Araújo, O., 1900. Diccionario geográfico de Uruguay. Imprenta Artística de Dornaleche y Reyes, Montevideo, Uruguay.

Balée, W., 1994. Footprints of the Forest: Kaapor Ethnobotany the Historical Ecology of Plant Utilization by an Amazonian People. Columbia University Press, New York.

Balée, W., 2013. Cultural Forests of the Amazon. University of Alabama Press, Tuscaloosa.

Bespalez, E., 2010. Levantamento arqueológico na aldeia Lalima, Miranda/MS: uma contribuição ao estudo da trajetória histórica da ocupação indígena regional. Revista de Arqueologia 23 (2), 118-146.

Bonomo, M., 2013. Reanálisis de la colección de Samuel Lothrop procedente del Delta del Paraná. Relaciones de la Sociedad Argentina de Antropología 38 (1), 169-198.

Bonomo, M., Politis, G., Gianotti, C., 2011. Montículos, jerarquía social y horticultura en las sociedades indígenas del Delta del río Paraná (Argentina). Latin American Antiquity 22 (3), 297-333.

Brochado, J.P., 1969. Pesquisas arqueológicas nos vales do Ijuí e do Jacuí. Publicações Avulsas do Museu Paranaense Emilio Goeldi 13, 31-62.

Brochado, J.P., 1971. Extensão das pesquisas arqueológicas nos vales do Jacuí e Ibicuí-Mirim, Rio Grande do Sul. Publicações Avulsas do Museu Paranaense Emilio Goeldi 15, 11-36.

Brochado, J.P., 1973. Migraciones que difundieron la tradición alfarera Tupí-Guaraní. Relaciones de la Sociedad Argentina de Antropología 7, 7-39.

Brochado, J.P., 1977. Alimentação na floresta tropical. Analogia etnográfica na reconstrução da alimentação por meio de evidências indiretas. Cadernos do Instituto de Filosofia e Ciências Humanas 2. Universidade Federal do Rio Grande do Sul Porto Alegre.

Brochado, J.P., 1984. An Ecological Model of the Spread of Pottery and Agriculture into Eastern South America (Ph.D. thesis). University of Illinois at UrbanaChampaign, Urbana, EEUU.

Brochado, J.P., 1989. A expansão dos Tupi e da cerâmica policrôma amazónica. Dédalo 27, 65-82.

Brochado, J.P., 1991. What did the Tupinambá cook in their vessels? A humble contribution to ethnography analogy. Revista de Arqueologia 6, 40-88.

Brochado, J.P., Monticelli, G., 1994. Regras Práticas na Reconstrução Gráfica das Vasilhas Guarani a Partir dos Fragmentos. Estudos Ibero Americanos 20 (2), 107-119.

Brochado, J.P., Monticelli, G., Neumann, E.S., 1990. Analogia Etnográfica na Reconstrução Gráfica das Vasilhas Guarani Arqueologicas. Veritas 35, 727-743.

Cabrera Pérez, L., 1994. La presencia Tupiguaraní en el Bajo Paraná y Uruguay durante los siglos XV y XVI. In: V Jornadas Internacionales Misiones Jesuíticas. Montevideo, pp. 333-344.

Cabrera Pérez, L., 2011. Patrimonio y arqueología en la región platense. Universidad de La Republica, Montevideo.

Caggiano, M.A., 1984. Prehistoria del Noreste argentino, sus vinculaciones con la República Oriental del Uruguay y sur de Brasil. Pesquisas, Antropología 38, $1-109$.

Castillo, A., 2004. Excavación y Museo: profundizando en el conocimiento de los grupos ceramistas del litoral (Río Negro, Uruguay). In: Beovide, L., Barreto, I., Curbelo, C. (Eds.), La Arqueología Uruguaya ante los desafíos del Nuevo Siglo. CD-ROM, Montevideo, Uruguay.

Carle, M.B., 2002. Investigação arqueológica em Rio Grande: uma proposta da ocupação Guarani pré-histórica no Rio Grande do Sul (Master thesis). Pontifícia Universidade Católica do Rio Grande do Sul, Porto Alegre, Brasil.

Ceruti, C.N., Crowder, R., 1973. La presencia de cerámica en los cordones conchiles litorales de la Provincia de Buenos Aires (Argentina). Un sitio nuevo. In: Anales de los Congresos Museo de Ciencias Naturales de Río Negro. Fray Bentos, Uruguay.

Chmyz, I., 1966. Terminologia arqueológica brasileira para a cerâmica. In: Manuais de Arqueologia. Centro de Ensino e Pesquisas Arqueológicas da Universidade Federal do Paraná, Curitiba, Brasil.

Chmyz, I., 1967. Dados parciais sobre a arqueologia do vale do rio Paranapanema. Publicações Avulsas do Museu Paranaense Emilio Goeldi 6, 59-78.

Chmyz, I., 1968. Novas perspectivas da arqueologia guarani no Estado do Paraná. Pesquisas Antropologia 18, 171-189.

Chmyz, I., 1969a. Dados parciais sobre a arqueologia do vale do rio Ivaí. Publicações Avulsas do Museu Paranaense Emilio Goeldi 10, 95-118.

Chmyz, I., 1969b. Pesquisas arqueológicas no Alto e Médio Iguaçu. Publicações Avulsas do Museu Paranaense Emilio Goeldi 13, 103-132.

Chmyz, I., Chmyz, J.C., 1986. Datações radiométricas em áreas de salvamento arqueológico do Estado do Paraná. Revista do CEPA 5, 69-77.

Cigliano, M.E., 1968. Investigaciones arqueológicas en el río Uruguay medio y la costa NE de la provincia de Buenos Aires. Pesquisas, Antropologia 18, 5-9.

Cigliano, E.M., Schmitz, P.I., Caggiano, M.A., 1971. Sitios cerámicos prehispánicos en la costa septentrional de la provincia de Buenos Aires y de Salto Grande, Entre Ríos. In: Esquema tentativo de su desarrollo, pp.131-191. Anales de la Comisión de Investigaciones Científicas de la provincia de Buenos Aires CXCII(II-III).

Corrêa, A.A., 2009. Tetama nas Matas Mineiras: sítios Tupi na microrregiao de Juiz de Fora- MG (Disertación). Museu de Arqueologia e Etnologia da Universidade de São Paulo, São Paulo, Brasil.

Correa da Silva, B.C., 2010. Etnolinguística e Etno-História Tupí: desfragmentando o olhar. Revista de Estudos da Linguagem 18 (1), 61-86.

Costa Angrizani, R., 2012. Variabilidad, Movilidad y Paisaje: una propuesta interpretativa para los vestigios de los asentamientos precoloniales en el noroeste 
del Rio Grande do Sul (Brasil) (Ph.D. thesis). Universidad Nacional de La Plata, La Plata, Argentina.

Dias, A.S., 1995. Um projeto para a arqueologia brasileira: breve histórico da implementação do Programa Nacional de Pesquisas Arqueológicas (PRONAPA). Revista do CEPA 19 (22), 25-39.

Diaz, A., 1977. Arqueología de Salto Grande: secuencia cultural resultante de las investigaciones realizadas en Isla de Arriba y del Medio (Uruguay). Actas del V Encuentro de Arqueología del Litoral 155-164 (Fray Bentos, Uruguay).

Evans, C., Meggers, B.J., 1965. Guia para Prospecção Arqueológica no Brasil. Guias 2. CNPq, INPA, MPEG, Belém.

Faccio, N., 1992. Estudo do Sítio Arqueológico Alvim no Contexto do Projeto Paranapanema (Master thesis). Universidade de São Paulo (USP), São Paulo.

Faccio, N., 1998. Arqueologia dos cenários das ocupações horticultoras de Capivara, baixo Parapanema (Ph.D. thesis). Museu de Arqueologia e Etnologiada Universidades de São Paulo, São Paulo, Brasil.

Farías Gluchy, M.E., 2005. El guaraní arqueológico meridional: entre el axioma y la heterodoxia (Ph.D. thesis). Pontificia Universidade Católica do Rio Grande do Sul, Porto Alegre, Brasil.

Farias, D.S.E., Kneips, A. (Eds.), 2010. Panorama Arqueológico de Santa Catarina. Editora Unisul, Palhoca.

Figini, A., 1993. Geocronología: métodos de datación en el Cuaternario. Laboratorio de Tritio y Radiocarbono, CONICET-UNLP, La Plata, Argentina.

Gaulier, P., 2001-2002. Ocupação pré-histórica Guarani no município de Porto Alegre, RS: considerações preliminares e primeira datação do sítio arqueológico RS-LC-71: Ilha Francisco Manuel. Revista de Arqueologia 14/15, 57-73.

Heckenberger, M.J., Neves, E.G., Petersen, J.B., 1998. De onde surgem os modelos? as origens e expansões Tupi na Amazônia Central. Revista de Antropologia 41 (1), 69-96.

Hilbert, K., 1999. Arqueologia guarani na região de Guaíba-RS. In: Resumos da X Reunião Científica da Sociedade de Arqueologia Brasileira. Recife, Brasil.

Hodder, I., 1979. Economic and social stress and material culture patterning. American Antiquity 44 (3), 446-454.

Hogg, A., Hua, Q., Blackwell, P.G., Niu, M., Buck, C.E., Guilderson, T.P., Heaton, T.J., Palmer, J.G., Reimer, P.J., Reimer, R.W., Turney, C.S.M., Zimmerman, S.R.H., 2013. SHCAL13 southern hemisphere calibration, 0-50,000 years CAL BP. Radiocarbon 55 (4), 1889-1903.

Iriondo, M.H., García, N.O., 1993. Climatic variations in the Argentine plains during the last 18,000 years. Palaeogeography, Palaeoclimatology, Palaeoecology 101, 209-220.

Kashimoto, E., 1997. Variáveis ambientais e arqueologia no Alto Paraná (Ph.D. thesis). Museu de Arqueologia e Etnologia da Universidade de São Paulo, São Paulo.

Kashimoto, E., Martins, G., 2008. A problemática arqueológica da Tradição cerâmica Tupiguarani no Mato Grosso do Sul. In: Prous, A., Lima, T.A. (Eds.), Os ceramistas tupiguarani. Sigma, Belo Horizonte, Brasil, pp. 149-178.

Klimek, S., Mielke, W., 1935. An analysis of the material culture of the Tupi peoples. American Anthropologist 37 (1), 71-91.

Kreutz, M.R., 2008. O contexto ambiental e as primeiras ocupações humanas do Vale do Taquari-RS (Master thesis). Centro Univérsitario UNIVATES, Lajeado.

La Salvia, F.L., Brochado, J.P., 1989. Cerâmica Guarani. Posenato Arte \& Cultura, Porto Alegre.

Lathrap, D.W., 1956. An archaeological classification of culture contact situations. In: Wauchope, R. (Ed.), Seminars in Archaeology, 1955, Society for American Archaeology Memoirs 11, pp. 3-30.

Lathrap, D.W., 1970. The Upper Amazon. Thames \& Hudson, London.

Lima, T.A., 2011. O problema de atribuição de identidades étnicas a registros arqueológicos. In: Loponte, D., Acosta, A. (Eds.), Arqueología Tupiguaraní. Instituto Nacional de Antropología y Pensamiento Latinoamericano, Buenos Aires, Argentina, pp. 7-21.

Lino, J.T., 2007. Arqueologia Guarani na bacia hidrográfica do rio Araranguá, Santa Catarina (Master thesis). Universidade Federal do Rio Grande do Sul, Porto Alegre.

Long, A., 1965. Smithsonian Institution radiocarbon measurements II. Radiocarbon 7, 245-256.

Long, A., Mielke, J., 1967. Smithsonian Institution radiocarbon measurements IV. Radiocarbon 6, 368-381.

Loponte, D., Acosta, A., Capparelli, I., Pérez, M., 2011. La arqueología guaraní en el extremo meridional de la cuenca del Plata. In: Loponte, D., Acosta, A. (Eds.), Arqueología Tupiguaraní. Instituto Nacional de Antropología y Pensamiento Latinoamericano, Buenos Aires, Argentina, pp. 111-154.

Lothrop, S.K., 1932. Indians of the Paraná Delta, Argentina. Annals of New York Academic Science 33, 77-232.

Maidment, D., 2002. Why Archydro. In: Maidment, D. (Ed.), Arc Hydro: GIS for Water Resources. ESRI Inc, Nueva York, pp. 55-86.

Marrero, A.R., Silva-Junior, W.A., Bravi, C.M., Hutz, M.H., Petzl-Erler, M.L., RuizLinares, A., Salzano, F.M., Bortolini, M.C., 2007. Demographic and evolutionary trajectories of the Guarani and Kaingang natives of Brazil. American Journal of Physical Anthropology 132, 301-310.

Martins, G.R., Kashimoto, E.M., Tatumi, S.H., 1999. Datações arqueológicas em Mato Grosso do Sul. Revista do Museu de Arqueologia e Etnologia 9, 73-93.

Meggers, B., Evans, C., 1970. Como interpretar a linguagem da cerâmica: manual para arqueólogos. Smithsonian Institution, Washington, D.C.

Meggers, B., Evans, C., 1983. Lowland South America and the Antilles. In: Jennings, J.D. (Ed.), Ancient South Americans. W. H. Freeman and Company, San Francisco, EEUU, pp. 286-335.
Meggers, B., Dias, O., Miller, E., Perota, C., 1988. Implications of archaeological distributions in Amazonia. In: Vanzolini, P., Heyer, W. (Eds.), Proceedings of a Workshop on Neotropical Distribution Patterns. Academia Brasileira de Ciências, Rio de Janeiro, pp. 275-294.

Melià, B., 1986. El guaraní conquistado y reducido. Ensayos de etnohistoria. CEPAG, Asunción del Paraguay.

Menghín, O.F.A., 1956. El Poblamiento Prehistórico de Misiones. Anales de Arqueología y Etnología 12, 19-40.

Métraux, A., 1927. Les migrations historiques des tribus Tupi-Guarani. Journal de la Société dés Américanistes 19, 1-45.

Métraux, A., 1928. La Civilisation matérielle des tribus Tupi-Guarani. Paul Geuthner, Paris.

Metz, M., Mitasova, H., Harmon, R., 2011. Efficient extraction of drainage networks from massive, radar-based elevation models with least cost path search. Hydrology and Earth System Sciences 15, 667-678.

Mielke, J., Long, A., 1969. Smithsonian Institution radiocarbon measurements V. Radiocarbon 11 (1), 163-182.

Milheira, R.G., 2008. Território e Estratégia de Assentamento Guarani na Planície Sudoeste da Laguna dos Patos e Serra do Sudeste (Master thesis). Museu de Arqueologia e Etnologia da Universidade de São Paulo, São Paulo.

Milheira, R.G., 2011. Os Guarani e seus artefatos líticos: um estudo tecnológico no sul do Brasil. Revista do Museu de Arqueologia e Etnologia 21, 129-152.

Milheira, R.G., De Blasis, P., 2011. O território guarani no litoral sulcatarinense: ocupação e abandono no limiar do período colonial. Revista de Arqueología Americana 29, 147-182.

Miller, E.T., 1967. Pesquisas arqueológicas efetuadas no nordeste do Rio Grande do Sul. Publicações Avulsas do Museu Paranaense Emílo Goeldi 6, 15-38.

Miller, E.T., 1969. Pesquisas arqueológicas efetuadas no noroeste do Rio Grande do Sul. Publicações Avulsas do Museu Paranaense Emílo Goeldi 10, 33-54.

Miller, E.T., 1971. Pesquisas arqueológicas efetuadas no Planalto Meridional, Rio Grande do Sul. Publicações Avulsas do Museu Paranaense Emílo Goeldi 15, $37-70$.

Mitas, L., Mitasova, H., 1999. Spatial interpolation. In: Longley, P., Goodchild, M.F., Maguire, D.J., Rhind, D.W. (Eds.), Geographical Information Systems: Principles, Techniques, Management and Applications. GeoInformation International, Wiley, New York, pp. 481-492.

Mujica, J.I., 1995a. Primeras aproximaciones sobre el uso del espacio abierto en una aldea guaraní prehispánica. In: Actas y Memorias del XI Congreso Nacional de Arqueología Argentina. San Rafael, Argentina, pp. 123-141.

Mujica, J.I., 1995b. De Corrientes Argentina. Informe de dos sitios arqueológicos guaraní en la provincia. In: XX Encuentro de Geohistoria Regional, Gobernador Virasoro, Argentina, pp. 119-127.

Mujica, J.I., 1995c. Un sitio guaraní en el centro de la provincia de Corrientes -Llamarada- Santa Rosa. Depto. de Concepción. In: XX Encuentro de Geohistoria Regional, Gobernador Virasoro, Argentina, pp. 135-148.

Naue, G., 1973. Dados sobre o estudo dos cerritos na área meridional da lagoa dos Patos, Rio Grande, Rio Grande do Sul. Revista Veritas 71 (3), 246-269.

Neves, W.A., Vicensotto Bernardo, D., Okumura, M., Ferreira de Almeida, T., Menezes Strauss, A., 2011. Origem e dispersão dos Tupiguarani: o que diz a morfologia craniana? Boletim do Museu Paraense Emílio Goeldi 6 (1), 95-122.

Nimuendajú, C., 1982. Mapa etno-histórico de Curt Nimuendajú. IBGE/Fundação Nacional Pró-Memória, Rio de Janeiro, Brasil.

Noelli, F.S., 1993. Sem tekohá não há tekó (em busca de um modelo etnoarqueológico da aldeia e da subsistencia Guarani e sua aplicação a uma área de domínio no delta do rio Jacuí-RS). Porto Alegre (Master thesis). Pontifícia Universidade Católica do Rio Grande do Sul, Porto Alegre.

Noelli, F.S., 1998. The Tupi: explaining origin and expansions in terms of archaeology and historical linguistics. Antiquity 72, 648-663.

Noelli, F.S., 1999-2000. A ocupação humana na Região Sul do Brasil: arqueologia, debates e perspectivas -1872-2000. Revista USP 44 (2), 218-269.

Noelli, F.S., 2004. La distribución geográfica de las evidencias arqueológicas guaraní. Revista Indias 64 (230), 17-34.

Noelli, F.S., 2008. José Proenza Brochado: vida acadêmica e Arqueologia Tupi. In: Prous, A., Andrade Lima, T. (Eds.), Os ceramistas Tupiguarani. Sínteses regionais Sigma/Sociedade de Arqueologia Brasileira/IPHAN, Belo Horizonte, pp. 17-47.

Noelli, F.S., Brochado, J.P., 1998. O cauim e as beberagens dos Guarani e Tupinambá: equipamentos, técnicas de preparação e consumo. Revista do Museu de Arqueologia e Etnologia 8, 117-128.

Noelli, F.S., Milheira, R.G., Wagner, G.P., 2014. Os Sítios arqueológicos Guarani do litoral sul do Brasil, Uruguai e Argentina. Registros até 2013. In: Milheira, R.G., Wagner, G.P. (Eds.), Arqueologia Guarani no litoral Sul do Brasil. Appris, Curitiba pp. 177-186.

Oliver, J.R., 1991. Donald W Lathrap: contributions to New World Archaeology. Antropológica 75-76, 5-60.

Oliver, J.R., 2008. The archaeology of agriculture in ancient Amazonia. In: Silverman, H., Isbell, W.H. (Eds.), Handbook of South American Archaeology. Springer, New York, pp. 185-216.

Olivera, F., Furnans, J., Maidment, D., Djokic, D., Ye, Z., 2002. Drainage systems. In: Maidment, D. (Ed.), Arc Hydro: GIS for Water Resources. ESRI Inc, New York, pp. 55-86.

Olaya Ferrero, V., 2004. Hidrologia Computacional y Modelos Digitales de Terreno. In: Creative Common Attribution Share-alike. http://www.gabrielortiz.com/ descargas/hidrologia _computacional_mdt_sig.pdf.

Outes, F.F., 1918. Nuevos rastros de la cultura guaraní en la cuenca del Paraná Inferior. Anales de la Sociedad Científica Argentina 85, 153-182. 
Pärssinen, M., 2003. When did the Guaraní expansion toward the Andean foothills begin?. In: Pärssinen, M., Korpisaari, A. (Eds.), Western Amazonia. Multidisciplinary Studies on Ancient Expansionistic Movements, Fortifications and Sedentary Life, vol. 14. Renvall Institute Publications, Helsinki, pp. 73-89.

Piazza, W., 1969. Notícia arqueológica do vale do Uruguai. Publicações Avulsas do Museu Paranaense Emílio Goeldi 10, 55-70.

Politis, G., Bonomo, M., 2013. La entidad arqueológica Goya-Malabrigo (Ríos Paraná y Uruguay) y su filiación Arawak. Boletín de la Sociedade de Arqueologia Brasileira 25 (1), 10-46.

Poujade, R.A., 1994. Mapa arqueológico de la provincia de Misiones. Universidad Nacional de Misiones, Entidad Binacional Yacyretá, Asunción, Paraguay.

Pronapa, Programa Nacional de Arqueologia Brasileira, 1970. Brazilian archaeology in 1968: an interim report on the national program of archaeological research. American Antiquity 35, 1-23.

Prous, A., Lima, T.A. (Eds.), 2008. Os ceramistas Tupiguarani. Sigma, Belo Horizonte.

Ramallo, V., Bisso-Machado, R., Bravi, C., Coble, M.D., Salzano, F.M., Hünemeier, T. Bortolini, M.C., 2013. Demographic expansions in South America: enlightening a complex scenario with genetic and linguistic data. American Journal of Physical Anthropoly 150, 453-463.

Ribeiro, P.A.M., 1968. Os sítios arqueológicos do vale do rio Caí. Pesquisas Antropologia 18, 153-169.

Ribeiro, P.A.M., 1974. Primeiras datações pelo método C14 para o vale do Caí, Rio Grande do Sul. Revista do CEPA 1, 16-22.

Ribeiro, P.A.M., 1991. Arqueologia do vale do rio Pardo, Rio Grande do Sul Brasil. Revista do CEPA 18 (21), 1-184.

Ribeiro, P.A.M., Ribeiro, C.T., Silveira, I., Klamt, S.C., 1986. Levantamentos arqueológicos nos altos vales dos rios Camaquã e Irapuá, RS, Brasil. Revista do CEPA 15, 41-70.

Rodrigues, A.D., 1964. A classificação do tronco lingüístico Tupi. Revista de Antropologia 12, 99-104.

Rodrigues, A.D., 1986. Línguas brasileiras. Para o conhecimento das línguas indí genas. Loyola, São Paulo, Brasil.

Rodrigues, A.D., 2007a. Agricultura Tupí Pré-Histórica na Amazônia. In: Resumos Congresso Internacional da Abralin, Belo Horizonte, p. 453.

Rodrigues, A.D., 2007b. As consoantes do Proto-Tupí. In: Cabral, A., Rodrigues, A.D. (Eds.), Línguas e Culturas Tupí. Curt Nimuendaju, Campinas, Brasília, pp. 167-203.

Rodrigues, A.D., 2010. Linguistic reconstruction of elements of prehistoric Tupí culture. In: Eithne, B., van der Kerke, S. (Eds.), Linguistics and Archaeology in the Americas: the Historization of Language and Society, pp. 1-10. The Netherlands, Leiden.

Rodrigues, A.D., Cabral, A.S., 2012. Tupían. In: Campbell, L., Grondona, V. (Eds.), The Indigenous Languages of South America. Mouton de Gruyter, Berlin/Boston, pp. 495-574.

Rodríguez, J.A., 1997. Investigaciones arqueológicas en Yacyretá (CorrientesArgentina). Jornadas de Antropología de la Cuenca del Plata 3, 41-47 (Rosario).

Rodríguez, J.A., 2009. La ocupación (poblamiento) del norte de Corrientes (Argentina) por fase de la Tradición Tupiguaraní. In: Meggers, B. (Ed.) Arqueologia interpretativa. O método quantitativo para o establecimento de sequências cerâmicas: estudos de casos. UNITINS, Porto Nacional, Brazil, pp. 49-62.
Rodríguez, J.A., Rodríguez, A.A., 1985. Proyecto Antropológico-Ecológico Salto Grande (Primer Informe). Universidad Nacional de Entre Ríos, Concordia, Argentina.

Rogge, J., 2005. Fenômenos de fronteira: um estudo das situações de contato entre portadores das tradições cerâmicas pré-históricas no Rio Grande do Sul. Pesquisas Antropologia 62, 9-119.

Rohr, J.A., 1969. Pesquisas arqueológicos do município sul-catarinense de Jaguaruna. Pesquisas Antropologia 22, 1-37.

Rusconi, C. 1928. Investigaciones arqueológicas en el sur de Villa Lugano (Capital Federal). Anales de la Sociedad Argentina de Estudios Geográficos 3 (1), 75-118.

Scatamacchia, M.C.M., 1990. A tradição policroma no leste da América do Sul evidenciada pela ocupação Guarani e Tupinambá: fontes arqueológicas e etnohistóricas (Ph.D. thesis). FFLeCH de la Universidade de São Paulo, São Paulo, Brasil.

Schmitz, P.I., 1991. Migrantes da Amazônia: a Tradição Tupiguarani. In: Kern, A.A. (Ed.), Arqueologia Pré-Histórica do Rio Grande do Sul. Mercado Aberto, Porto Alegre, Brasil, pp. 295-330.

Schmitz, P.I., Brochado, J.P., 1972. Datos para una secuencia cultural Del Estado de Rio Grande do Sul, Brasil. In: Gabinete de Arqueologia 2. Universidade Federal do Rio Grande do Sul, Porto Alegre, Brasil.

Schmitz, P.I., Sandrin, C., 2009. O sítio Lagoa dos Índios e o povoamento guarani da planície costeira do Rio Grande do Sul. Documentos 11, 89-134.

Sempé, M.C., Caggiano, M.A., 1995. Las culturas agroalfareras del Alto río Uruguay (Misiones). Argentina. Revista do Museu de Arqueologia e Etnologia 5, 27-38.

Silva, F.A., 2008. Ceramic technology of the Asurini do Xingu, Brazil: an ethnoarchaeological study of artifact variability. Journal of Archaeological Method and Theory 15, 217-265.

Soares, A.L.R., 2001-2002. Arqueologia, história e etnografia: o denominador Guarani. Revista de Arqueologia vol. 14/15, 97-114.

Stevaux, J.C., 2000. Climatic events during the late Pleistocene and Holocene in the upper Parana River: correlation with NE Argentina and South-Central Brazil. Quaternary Intrernational 72, 73-86.

Storto, L.R., Moore, D., 2001. As Línguas Indígenas e a Pré-História. In: Pena, S. (Ed.), Homo brasilis: Aspectos Genéticos, Linguísticos, Históricos e Sócio-antropológicos da Formação do Povo Brasileiro. FUNPEC, Ribeirao Preto.

Stuckenrath, R., Mielke, J., 1970. Smithsonian Institution radiocarbon measurements VI. Radiocarbon 12 (1), 193-204.

Stuckenrath, R., Mielke, J. 1972. Smithsonian Institution radiocarbon measurements VII. Radiocarbon 14 (2), 401-412.

Stuckenrath, R., Mielke, J., 1973. Smithsonian Institution radiocarbon measurements VIII. Radiocarbon 15 (2), 388-424.

Stuiver, M., Reimer, P.J., Reimer, R., 2013. CALIB 7.0.0. Www Program and Documentation. http://calib.qub.ac.uk/calib/.

Urban, G. 1992. A história da cultura brasileira segundo as línguas nativas. In: da Cunha, M.C. (Ed.), História dos índios no Brasil. Cia das Letras, São Paulo, pp. $87-102$.

Vignati, M.A., 1941. Censo de paquetes óseos de origen guaraní. Revista del Museo de La Plata 2 (9) 1-11.

Willey, G.R., Phillips, P., 1958. Method and Theory in American Archaeology. University of Chicago Press, Chicago. 